University of South Florida

DIGITAL COMMONS

Digital Commons @ University of

@ UNIVERSITY OF SOUTH FLORIDA

South Florida

$11-11-2003$

\title{
Three Dimensional Finite Element Model for Lesion Correspondence in Breast Imaging
}

Yan Qiu

University of South Florida

Follow this and additional works at: https://digitalcommons.usf.edu/etd

Part of the American Studies Commons

\section{Scholar Commons Citation}

Qiu, Yan, "Three Dimensional Finite Element Model for Lesion Correspondence in Breast Imaging" (2003). USF Tampa Graduate Theses and Dissertations.

https://digitalcommons.usf.edu/etd/1452

This Thesis is brought to you for free and open access by the USF Graduate Theses and Dissertations at Digital Commons @ University of South Florida. It has been accepted for inclusion in USF Tampa Graduate Theses and Dissertations by an authorized administrator of Digital Commons @ University of South Florida. For more information, please contact digitalcommons@usf.edu. 
Three Dimensional Finite Element Model for Lesion Correspondence in Breast Imaging

by

\title{
Yan Qiu
}

\author{
A thesis submitted in partial fulfillment \\ of the requirements for the degree of \\ Masters of Science in Computer Science \\ Department of Computer Science and Engineering \\ College of Engineering \\ University of South Florida
}
Co-Major Professor: Dmitry Goldgof, Ph.D.
Co-Major Professor: Lihua Li, Ph.D.
Dmitry Goldgof, Ph.D.
Lihua Li, Ph.D.
Sudeep Sarkar, Ph.D.

Date of Approval:

November 11, 2003

Keywords: finite element model, mammogram, registration, correspondence, MRI

(C) Copyright 2003, Yan Qiu 


\section{Acknowledgments}

I gratefully acknowledge the help of Dr. Dmitry Goldgof and Dr. Lihua Li, who have supervised my thesis work and guided me through all steps of research and writing. I am grateful to Dr. Sudeep Sarkar for his valuable guidance and feedback. I also thank Sorin Anton and Yong Zhang for their comments and suggestions.

I have received invaluable support from my wife, Yu Huang, who has provided help and encouragement through my graduate studies. 


\section{Table of Contents}

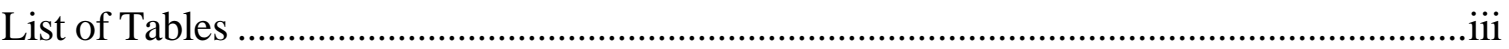

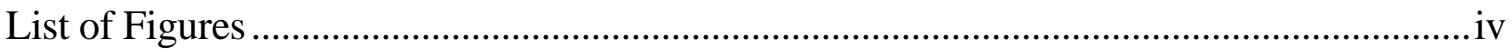

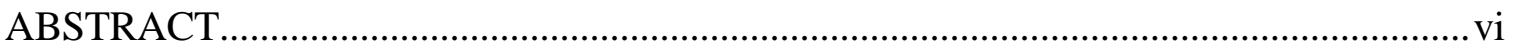

Chapter 1 Introduction ............................................................................................. 1

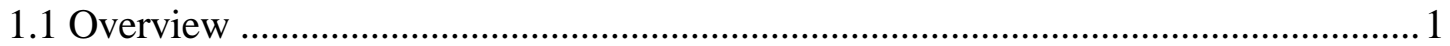

1.2 Previous Work and Pilot study .............................................................................

1.3 Elastic Theory .....................................................................................................

Chapter 2 Theoretical Background .................................................................................12

2.1 Breast Imaging................................................................................................... 12

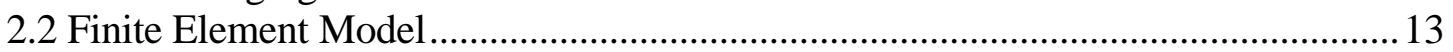

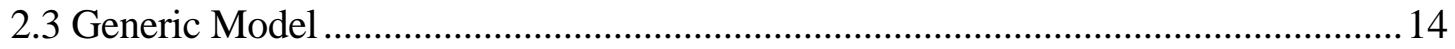

2.4 Breast Model Compression Simulation ...................................................................... 15

2.5 Material Properties ...........................................................................................

2.6 Meshing and Discretization ..................................................................................... 19

2.7 Registration............................................................................................................ 22

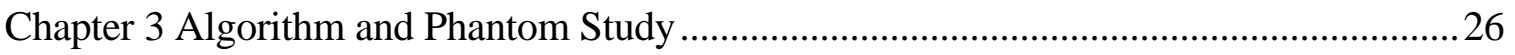

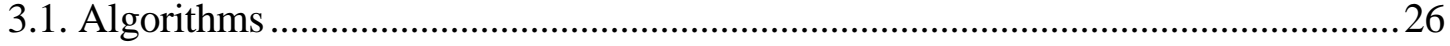

3.1.1 Algorithm Based on Minimum Euclidean Distance ..........................................2. 26

3.1.2 Algorithm for Curve Prediction.....................................................................2

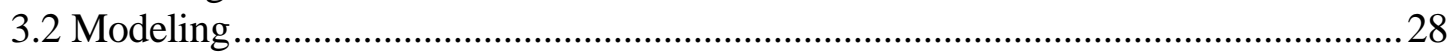

3.2.1 Image Acquisition and Data Extraction ........................................................2.28

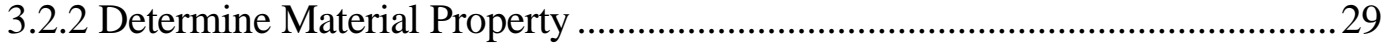

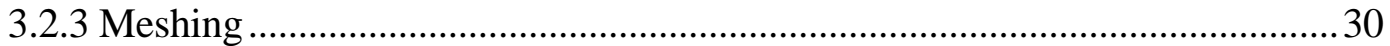

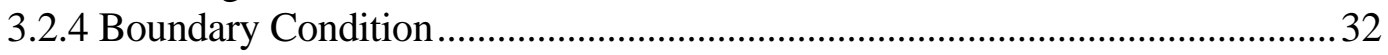

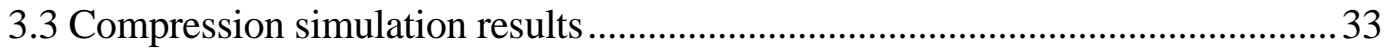

3.4 Validation .........................................................................................................

3.4.1 Validation Based on Minimum Euclidean Distance..............................................34

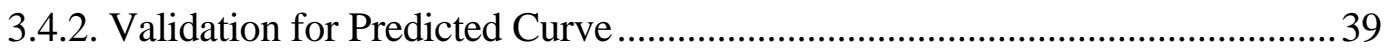

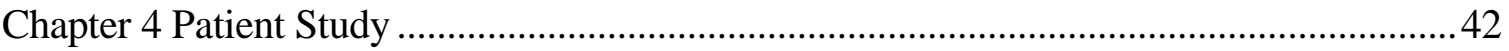

4.1 Image Acquisition and Data Extraction...................................................................42

4.2 Patient Generic Model................................................................................................4

4.3 Validation Based on Minimum Euclidean Distance ..................................................... 44

4.4 Validation for Predicted Curve ................................................................................... 45

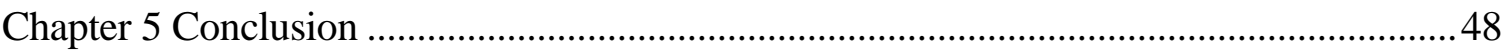




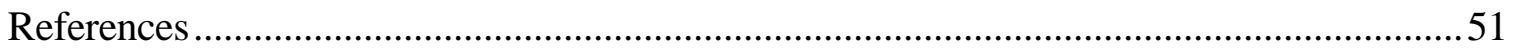




\section{List of Tables}

Table 1. Error Comparison for Different Models for Young's Modulus .............................19

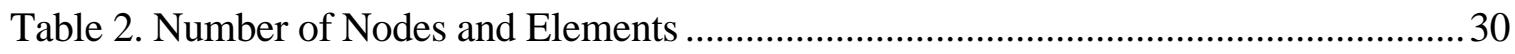

Table 3. Compression Values Used in X-ray Imaging ....................................................... 33

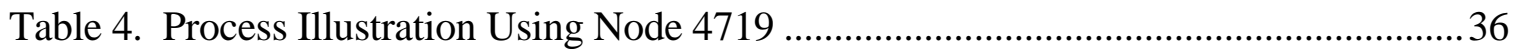

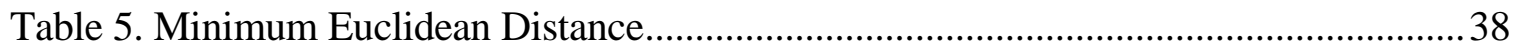

Table 6. Minimum Euclidean Distance Experiment Results for Patient Data...................... 45 


\section{List of Figures}

Figure 1. Compression of Mammogram in Two Views.........................................................12

Figure 2. Phantom Finite Element Model Illustration...............................................................13

Figure 3. Phantom Finite Element Generic Model Illustration ................................................ 14

Figure 4. Compression in the CC View to Match Mammogram ..............................................15

Figure 5. Finding Correspondent Lesion Elements in Two Views .........................................16

Figure 6. Tetrahedral Elements and Hexahedral Elements - ANSYS...................................21

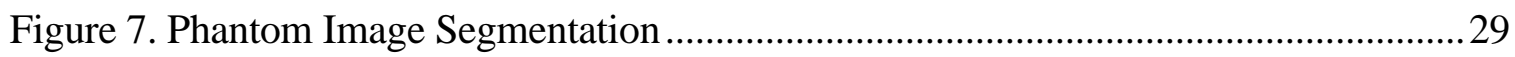

Figure 8. Model before and after meshing ........................................................................... 31

Figure 9. Finite Element Model Elements and Nodes...............................................................32

Figure 10. Finite Element Model Elements and Nodes............................................................33

Figure 11. Phantom CC view compression simulation ............................................................34

Figure 12. Phantom ML View Compression Simulation ......................................................34

Figure 13. Validation Using Minimum Euclidean Distance ...................................................... 35

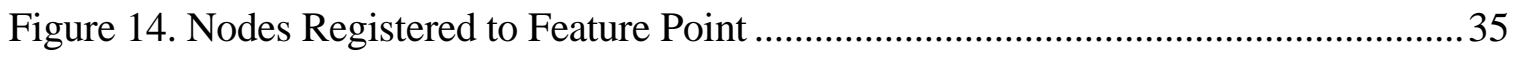

Figure 15. Simulated Calcification with Predicted Curve .......................................................37

Figure 16. Validation for Predicted Curve............................................................................. 39

Figure 17. Predicted Lesion Position in Phantom ML View ..................................................40

Figure 18. Patient MR Images........................................................................................ 42 
Figure 19. Segmented MRI Images after Morphological Operation ...................................42

Figure 20. Generic Model Scaling According to CC View and ML View ..........................43

Figure 21. Patient Data with Visible Lesion in Both Views ............................................ 45

Figure 22. Predicted Lesion Position in Patient Data ML View ........................................4 46 


\title{
THREE DIMENSIONAL FINITE ELEMENT MODEL FOR LESION CORRESPONDENCE IN BREAST IMAGING
}

\section{Yan Qiu}

\begin{abstract}
Predicting breast tissue deformation is of great significance in several medical applications such as surgery, biopsy and imaging. In breast surgery, surgeons are often concerned with a specific portion of the breast, e.g., tumor, which must be located accurately beforehand. Also clinically it is important for combining the information provided by images from several modalities or at different times, for the planning and guidance of interventions. Multi-modality imaging of the breast obtained by mammography, MRI and PET is thought to be best achieved through some form of data fusion technique. However, images taken by these various techniques are often obtained under entirely different tissue configurations, compression, orientation or body position. In these cases some form of spatial transformation of image data from one geometry to another is required such that the tissues are represented in an equivalent configuration.

We constructed the 3D biomechanical models for this purpose using Finite Element Methods (FEM). The models were based on phantom and patient MRIs and could be used to model the interrelation between different types of tissue by applying displacements of forces and to register multimodality medical images.
\end{abstract}




\section{Chapter 1 \\ Introduction}

\subsection{Overview}

Breast cancer is the second leading cause of cancer death for all women (after lung cancer), and the leading overall cause of cancer death in women between the ages of 40 and 59. In 2002, 257,800 new cases of breast cancer will be diagnosed, and 39,600 women will die from the disease. The risk of developing breast cancer seems to depend on several factors including age, personal or family history of breast cancer, parity, age at first birth, hormonal replacement, etc. However, over $70 \%$ of cancer cases are women with no identifiable risk factors. Early diagnosis is very important for proper treatment and cure and this has led many countries, including US, to develop regular screening programs that are primarily based on mammography and physical examination.

Mammography is the main screening tool for breast cancer with a sensitivity of about $85 \%$ and specificity up to $25 \%$. Despite their proven effectiveness, both screening tools entail significant variability and there are few attempts to-date to standardize either one or correlate mammographic to physical examination findings. Techniques that improve the accuracy of mammography or physical breast examination or both are still highly desirable and could benefit breast cancer diagnose. Furthermore, methodologies that yield a 3D representation of the breast with accurate volume and lesion location are 
expected to offer a unique tool for accurate and consistent follow-up, for correlation between findings from different screening procedures, as well as correlation of serial examinations (annual or serial exams).

Several approaches have been investigated until now to improve the diagnostic accuracy of mammography including computer-aided detection and diagnosis, computer displays, new direct digital mammography systems, and, recently, digital 3D mammography or tomosynthesis. Excluding tomosynthesis research, all other studies todate have been focused on the reconstruction of stereotactic biopsy images, the use of liquid crystal display glasses to create a 3D perception of the breast from monitors, and the combination of the 2D mammographic views using warping techniques to establish a degree of correlation and stereo representation. We have implemented several published methods from the latter class of methodologies in an effort to correlate the mammographic views and generate a 3D simulation of the compression.

Screening mammograms usually consist of a craniocaudal (CC) and mediolateral oblique (ML) view of each breast. Breast x-rays show areas of fatty and glandular tissue, pectoral muscle (if the view is ML), skin boundary, nipple and the non-breast region. Due to variation in compression and physical changes of the breast, consecutive mammograms of the same patient are difficult to fully correlate from one examination to the next and the expert reader may identify only general similarities. Similarly, due to differences in compression geometry and lack of common, reference points or fixed landmarks other than the nipple, one-to-one correspondence between the mammographic views is nearly impossible, and well-known stereo imaging algorithms widely used in stereo navigation, such as stereovision or passive ranging, cannot be applied to mammography. 
The goal of this thesis is to construct solid model for human breast, and design solid model-based methods to estimate non-rigid registration and non-rigid motion attributes, that is, to predict physical deformation and displacements of the breast and perform a nonrigid registration between two different X-ray views. This is useful for surgical procedures and diagnoses purposes. A 3D finite element model of the breast was constructed based on the MR slice images. Once we had the FEM model, a compression similar to one performed during mammography data acquisition, was simulated and a registration between the projected image of 3D volume and X-ray images was performed. The commercial software ANSYS was used to compute the FEM of the breast solid model.

\subsection{Previous Work and Pilot study}

There are many cases where the rigid body motion paradigm is inadequate. For instance most of the biological objects are flexible and articulated. In order to describe these types of deformations one must model the physics by which these objects deform. Underlying equations describing rigid motion and methods for solving them has been studied thoroughly [35][49].

In [47] and [48] the authors investigated the influence of different tissue elasticity values, Poisson ratios, boundary conditions, finite element solvers and mesh resolutions on a data set. MR images were acquired before and after breast compression. Images were aligned using a 3D non-rigid registration algorithm [37]. Biomechanical model of the breast was constructed using FEM. Two geometrical models were used in this study with different geometric resolutions. For the first, coarser geometric model, the segmented images were blurred by a Gaussian kernel and resampled to an $8 \mathrm{~mm}$ voxel size, and after 
the triangulation of the surface, the number of elements was decimated by 120 iterations. After meshing, these led to a model that had 51072 nodes and 34873 elements for fat and 4484 surface shells for skin. The second model was less coarse and resulted in 102102 nodes and 72756 elements, and 4950 surface shells. Five material models were used in this study:

- breast assumed linear and homogenous, 1KPa Young modulus was assigned to the mixture of glandular and fatty tissues;

- an additional Young's modulus of 88KPa was chosen for the skin;

- in addition to previous material model a 10KPa Young modulus was used to model separately the glandular tissue;

- non-linear material model [2], exponential curves were used to describe the stressstrain curves, Young modulus of fat increases linearly with strain from zero up to the value for glandular tissue;

- non-linear model proposed in [38], instead of exponential curves to describe stress-strain curves, quadratic and third order polynomials were used for fatty and glandular tissue respectively.

Four sets of boundary conditions were used:

- all surface nodes were constrained to the corresponding displacements obtained from 3D non-rigid registration;

- a subset of the previous nodes was used, which is the posterior, medial and lateral side of the breast;

- the nodes on the wall chest boundary were assigned to have zero displacement and no boundary conditions to other nodes; 
- besides the previous conditions, the nodes on the medial side of the breast were assigned to have zero displacement.

In ANSYS both the "Frontal Solver" and "Sparse Direct" solvers are direct elimination solvers and are recommended when robustness is required. The "Preconditioned Conjugate Gradient" is recommended for large solid models. All three solvers were used on different models. The conclusions of this test were that boundary conditions and the value of Poisson ratio have a much larger effect on the performance of the FEM model than using different tissue properties, although models with accurate boundary conditions seemed not to be much influenced by Poisson ratio. The mesh resolution and choice of finite element solver had almost no effect on the performance of the FEM of the breast.

In [16] six similarity measures are compared for use in intensity based 2D-3D image registration. The accuracy of each registration method is compared to a gold standard which was calculated using a feature-based algorithm. Similarity measures were used to register a fluoroscopic image and a digitally reconstructed radiograph. The similarity measures used are:

- normalized cross correlation;

- entropy of the difference of the image, the entropy measures operate on the difference image obtained by subtracting the scaled DRR image from fluoroscopy image;

- mutual information or relative entropy, it has been found very effective in 3D-3D multimodality image registration

- gradient correlation, the cross correlation is computed on the gradient images obtained using Sobel kernel; 
- pattern intensity also operates on difference image like entropy, but count the number of patterns in difference image that should tend to zero for a perfect registration - gradient difference involves the difference of the gradient images

The study concluded that the least accurate similarity measure for this experiment was mutual information. Gradient correlation has been shown to be sensitive to thin line structures. On images that contained both soft tissue and a stent cross correlation, entropy and mutual information failed often, and gradient correlation was the most accurate. Pattern intensity, gradient correlation and gradient difference were affected very little by the presence of the soft tissue.

In [14] three 3D-2D registration modalities were addressed for CT scan images and X-ray images. Here is presented the framework for finding a geometric transformation between a 3D image and a 2D radiography - 3D-2D curve registration problem, 3D-3D surface rigid registration by using passive stereo to reconstruct a 3D surface, and 3D-2D surface registration by using silhouettes for finding the transformation for blood vessels. In order to find an initial estimate of the projective transformation, the authors used the bitangent-line properties of the curves. For 3D-3D surface rigid registration, a passive stereo system was used and resulted in a dense description of the surface using points and normals. For 3D-2D projective surface registration, the authors used a combinatorial approach to find the initial estimate based on the property: "If a point $\mathrm{M}$ on a 3D surface $\mathrm{S}$ is such that its projection $m=\operatorname{Proj}(\mathrm{M})$ lies on the occluding contour $\mathrm{c}$, then the normal vector $\mathrm{N}$ to $\mathrm{S}$ at point $\mathrm{M}$ is equal to the normal vector $\mathrm{n}$ of the plane $\mathrm{P}$ defined by $(\mathrm{m}, \mathrm{O}, \mathrm{t})$, where $\mathrm{t}$ is the tangent vector to the occluding contour at point $\mathrm{m}$ ”. 
In [42] and [43] non-rigid registration methods were explored and in [9] a novel validation method was proposed. The method is based on finite element modeling of the breast in order to simulate plausible breast deformations. The model similar to [31] used the value of the Young modulus $1 \mathrm{KPa}$ for fatty tissue, $16.5 \mathrm{KPa}$ for the carcinoma and 88KPa for the skin. The registration was performed for the real compress breast image and the simulated image of the breast using FEM. The average error was about $1 \mathrm{~mm}$, with the minimum values as low as $0.08 \mathrm{~mm}$ in tumorous tissue and $0.15 \mathrm{~mm}$ in the overall tissue. In [43] the FEM method was used for validation for the registration technique using multiresolution with free-form deformations (FFD), based on multilevel B-splines and nonuniform control point distribution.

In [2] the authors developed a procedure to predict the displacement by plate compression of the breast that takes less than half an hour, making it clinically practical. In this study, they used the FEM of the deformable breast for guiding breast biopsy with MR imaging and registration between different breast MR data sets from the same patient, obtained at different times and at different compressions. Volume elements used in this finite element model of the breast were: hexahedral trilinear isoparametric elements to model the breast tissue and three-node triangular isoparametric elements to model the skin. All elements assumed to have nonlinear elastic material properties, isotropic, homogenous and incompressible. For each element after each deformation increment, the stiffness value of every element is updated to model the nonlinear behavior of material. The Young modulus for the fat tissue was assumed to have a quadratic behavior with respect to stiffness in order to try to take into account the Cooper ligaments. Only 8 MRI slices were stacked in order to obtain the 3D breast volume for the compression experiment and 58 for 
registration experiment. The results showed that the performance of the model was modestly affected by the material properties, but the shape and size of the patient breast, and the boundary properties between breast and plates have a great influence.

Sorin Anton [1] described Finite Element Model using different material property and boundary condition in his thesis. During my graduate work I further used Finite Element Model for phantom and patient study.

\subsection{Elastic Theory}

Non-rigid motion has been continuously studied in the last years and a variety of approaches had been presented, but until now no one paradigm can be applicable to all types of non-rigid motions.

Active contours were used to track motion. Active contours are a minimization problem and have been largely used. The general concept and one of its applications are presented in [27]. An insightful view of the underlying mathematics and a new algorithm for detecting objects which do not have boundaries defined by gradient is presented in [9]. In [19] the authors used the active contours to track the endocardial and epicardial borders of the left ventricle. In [51] the authors used active contours to track feature points between two images. In [15] is proposed a new method to incorporate prior shape information into geometric active contours for contour detection. Unlike traditional active contours (snakes) that are represented as parameterized curves in a Lagrangian formation, geometric active contours are represented as level sets of two dimensional distance functions which evolve according to an Eulerian formation. Active contours enforce constraints on smoothness and the amount of deformation, but they cannot be used to constrain the types of deformation 
valid for a particular object class. To overcome this problem apriori constraints must be enforced on the types of allowable deformations [41] [12].

Finite element models include material properties of the object and an underlying geometry model and can accurately predict displacements and motion based on applied forces, or recover the loads given nodal displacements. In [28] authors used FEM model to compute elastic properties of the scars relative to the surrounding area. In [51] the authors used FEM to recover elastic properties of the skin by incrementally modifying the material properties until the model matches the image of the deformed object. In [51] the authors used finite element model to compute intermediate images given images at two time instances and their corresponding features. In [52] the authors used FEM based algorithm for accurate non-rigid motion tracking. They used the difference between the actual behavior from the motion images and predicted behavior of the object in order to refine the model, and unknown parameters are recovered during the search for the best finite element model that approximates the non-rigid movement of a given object.

The dynamics of the elastic body is governed by the following system of partial differential equations:

$$
\begin{aligned}
& \rho_{0} \frac{\partial^{2} u}{\partial t^{2}}=(\lambda+\mu)\left(\frac{\partial^{2} u}{\partial x^{2}}+\frac{\partial^{2} v}{\partial y \partial x}+\frac{\partial^{2} w}{\partial z \partial x}\right)+\mu \nabla^{2} u+f_{x} \\
& \rho_{0} \frac{\partial^{2} v}{\partial t^{2}}=(\lambda+\mu)\left(\frac{\partial^{2} u}{\partial x \partial y}+\frac{\partial^{2} v}{\partial y^{2}}+\frac{\partial^{2} w}{\partial z \partial y}\right)+\mu \nabla^{2} v+f_{y} \\
& \rho_{0} \frac{\partial^{2} w}{\partial t^{2}}=(\lambda+\mu)\left(\frac{\partial^{2} u}{\partial x \partial z}+\frac{\partial^{2} v}{\partial y \partial z}+\frac{\partial^{2} w}{\partial z^{2}}\right)+\mu \nabla^{2} w+f_{z}
\end{aligned}
$$

where: $(u, v, w)$ represents the displacement vector in Cartesian coordinates 
E representsYoung's modulus

$v$ represents Poisson's ratio

$f_{i} \quad$ represents force field

$\mu, \lambda$ are Lame constants, computed with the formulas:

$$
\begin{gathered}
\mu=\frac{E}{2(1+v)} \\
\lambda=\frac{v E}{(1+v)(1-2 v)}
\end{gathered}
$$

For small deformations The Young's modulus can be considered constant and the deformation elastic. However in medical imaging large deformations are desirable to maximize the signal to noise ratio. At large deformations biological tissues will have more or less strain hardening, depending on tissue property. In our case for breast compression which undergoes significant deformation we can assume Young's modulus constant and equal to an average value of the initial and final state. In order to describe the deformation in response to an external solicitation, a tissue can be considered as isotropic and linear continuous elastic medium. In this case the relation between strain and stress can be expressed in tensor notation:

$$
\sigma_{i j}=2 \mu \varepsilon_{i j}+\lambda \delta_{i j} \varepsilon_{n n}
$$

where

$$
\begin{aligned}
& \sigma_{i j} \text { represents the symmetric stress tensor } \\
& \varepsilon_{i j} \text { represents the symmetric strain tensor } \\
& \delta_{i j} \text { represents the Kroneker delta defined as: }
\end{aligned}
$$




$$
\delta_{i j}=\left\{\begin{array}{l}
1, \text { if } i=j \\
0, \text { if } i \neq j
\end{array} \text { and } \varepsilon_{n n}=\sum_{i=1}^{3} \sum_{j=1}^{3} \varepsilon_{i j}\right.
$$

and for $i=j$

$$
\varepsilon_{n n}=\varepsilon_{11}+\varepsilon_{22}+\varepsilon_{33}
$$

In order to have a solution and to be unique for the system of partial differential equations we must have some boundary conditions (the initial conditions for the partial differential equations). The mechanical boundary conditions are given by [20]:

$$
\left(\sigma_{i j} n_{j}-F_{i}\right) \delta\left(u_{i}-u_{i}^{0}\right)=0
$$

where:

$n_{j}$ is the $\mathrm{j}^{\text {th }}$ component of the unit normal vector at the body surface

$F_{i}$ is the force per unit area at the surface acting in direction $x_{i}$

$u_{i}^{0}$ is the initial surface displacement

$\delta$ is a variation symbol 


\section{Chapter 2}

\section{Theoretical Background}

\subsection{Breast Imaging}

In X-ray imaging breast compression is necessary to flatten the breast so that the maximum amount of tissue can be imaged, and to reduce x-ray scatter which leads to image degradation. Usually two or more X-rays are taken on different angles, with different compression values for each angle of view. To match the features from one view to the feature in another view is not a trivial task and usually is done by a trained physician.

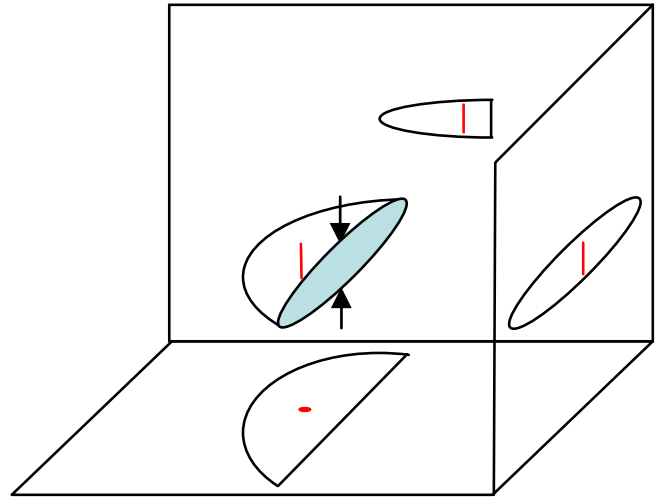

(a)Compression of Mammogram in CC View

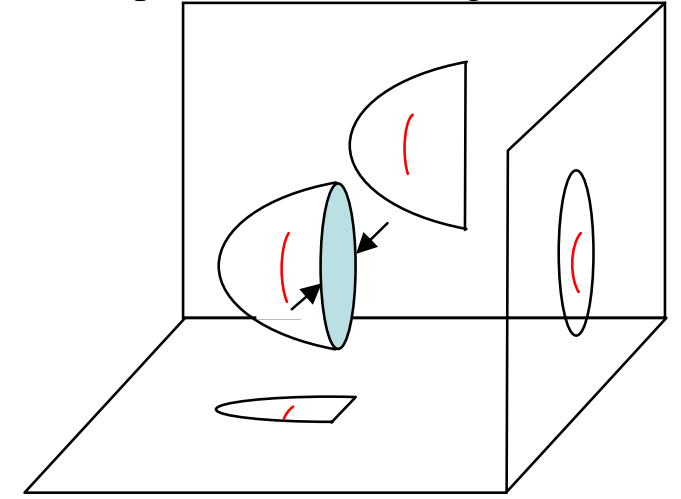

(c) Compression of mammogram in ML view

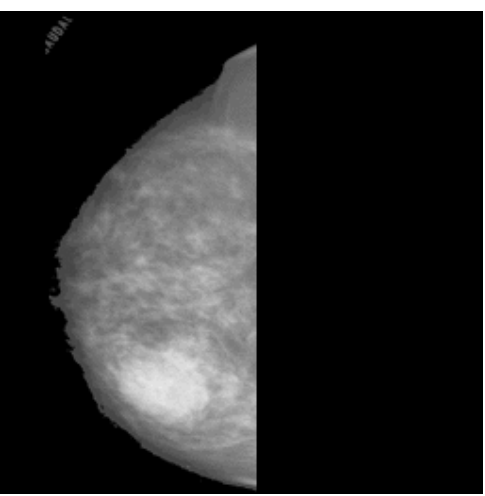

(b)Mammogram in CC View

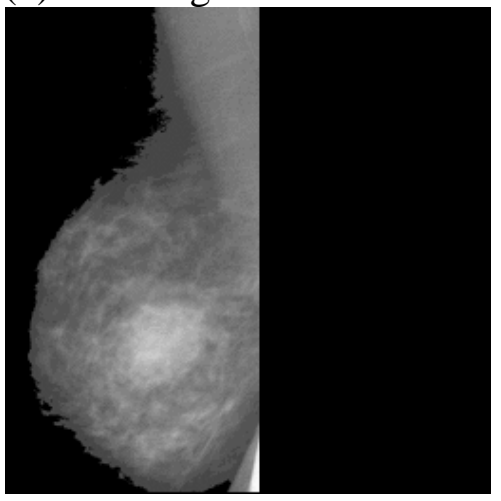

(d) Mammogram in ML view Figure 1. Compression of Mammogram in Two Views 


\subsection{Finite Element Model}

The finite element model approach is based on the underlying geometry of the object and on the material properties of the object. Using a system of partial differential equations to predict the movement of each node, shape analysis of the constitutive elements in each state, material properties and a set of border conditions to insure the convergence of the solution, FEM can predict with high accuracy the final state of the object, or any intermediate state.

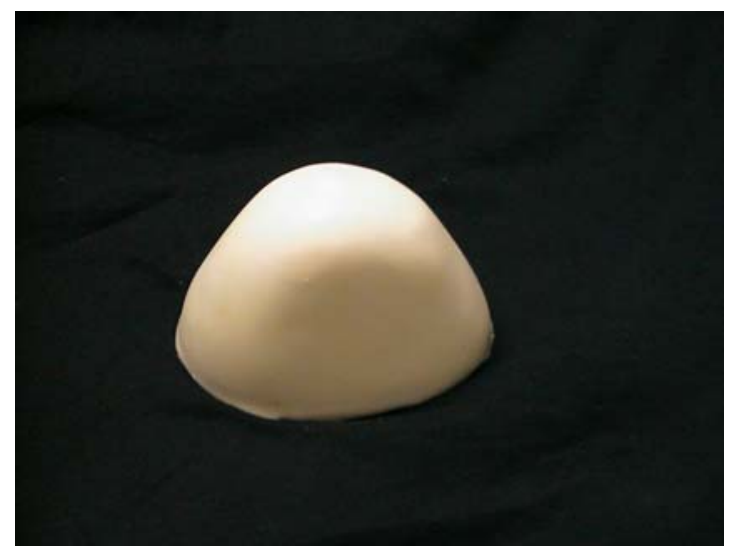

(a) Digital image of phantom

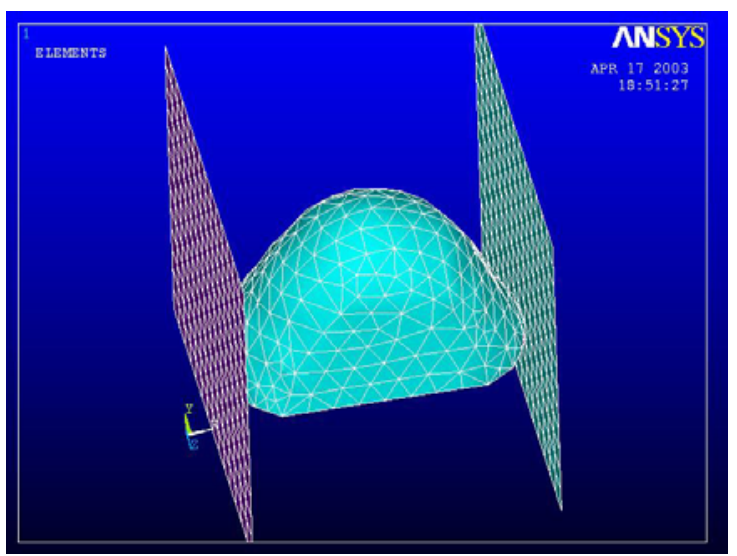

(b) Phantom model after meshing

Figure 2. Phantom Finite Element Model Illustration

Biomechanical models of the breast are being developed for a wide range of applications including image alignment tasks to improve diagnosis and therapy monitoring, imaging related studies of the biomechanical properties of lesions, and image guided interventions. From the need to register features in different views of the same organ, or the need to know what displacement will have a particular tissue under external controlled applied deformations emerged the necessity to have a 3D representation of the object and to be able to predict deformations and/or strain distributions inside the object during external deformations. The necessity for a non-rigid registration between two different 
views inspired the first part of this study. The modeling concepts also hold for the second part of the study, but the goal is different.

\subsection{Generic Model}

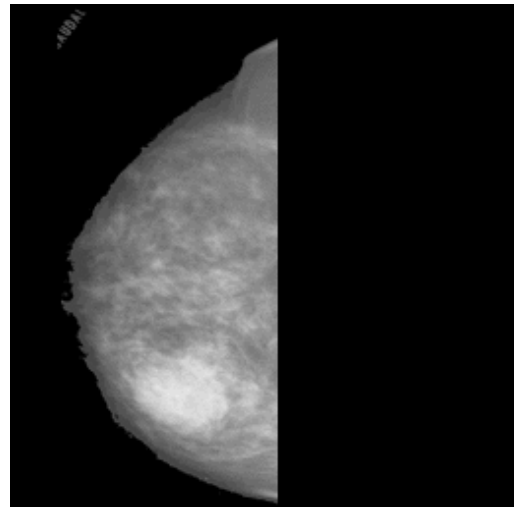

(a) CC View X-ray

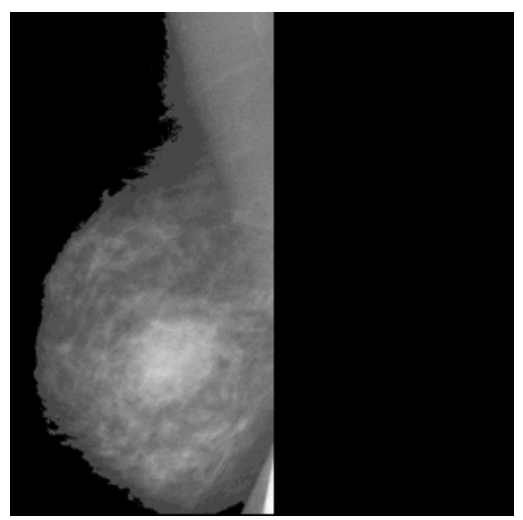

(c) ML View X-ray

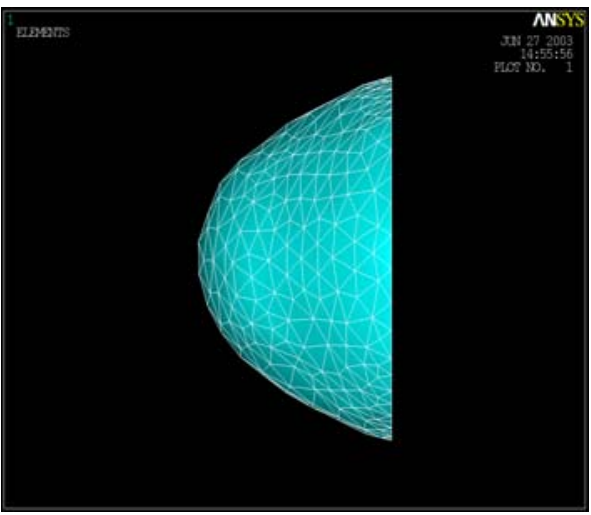

(b) Generic Model Scaled According to CC View

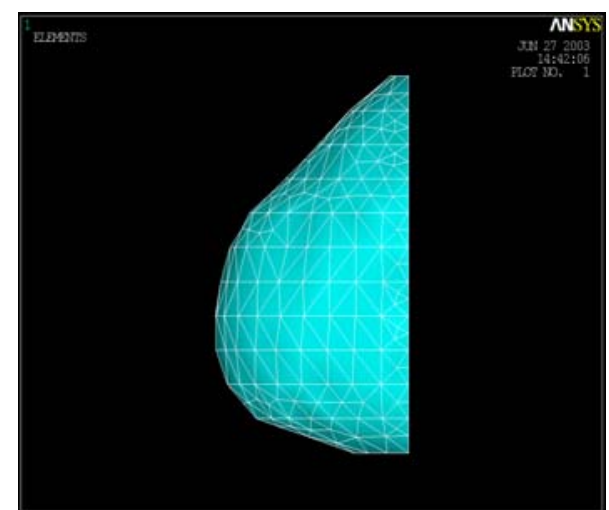

(d) Generic Model Scaled According to ML View

Figure 3. Phantom Finite Element Generic Model Illustration

In our experiments using patient data, generic model was used in the case when no MRI from the patient is available. The first step is to build a generic breast model based on well segmented MR images for one breast and apply this generic model for registration between X-ray images for different breast. For this method to work, the generic model must be scaled first and after that attempt the registration. The generic model was first compressed and projected in CC and ML views. The $\mathrm{x}$ and $\mathrm{y}$ scaling factors were obtained by scaling the projected CC view along $\mathrm{x}$ and $\mathrm{y}$ axis to match the 
X-ray image. These scaling factors were then taken into the consideration when we simulated the breast compression. Using these $\mathrm{x}$, $\mathrm{y}$ scaling factors we recomputed the generic model and use it for registration between the two X-ray views. The results for matching the scaled model with the ML X-ray view are presented as follows:

\subsection{Breast Model Compression Simulation}

In order to be able to match a feature from the X-ray CC view to X-ray ML view, first the breast model is compressed along the $\mathrm{z}$ direction with a displacement value identical to X-ray compression. To solve the system of partial differential equations, a sparse method solver and a preconditioned conjugate gradient (PCG) with full NewtonRaphson iterative method were used. The PCG method performs better for solid model which has a large number of elements. After computing the predicted displacement of the nodes, the 3D compressed breast model was projected on XY plane perpendicular to the compression direction, resulting a 2D image that should match the X-ray CC view. The next step involved in experiment was to register the projected image and the CC X-ray image, matching the feature pixels from CC X-ray image to projected node positions.

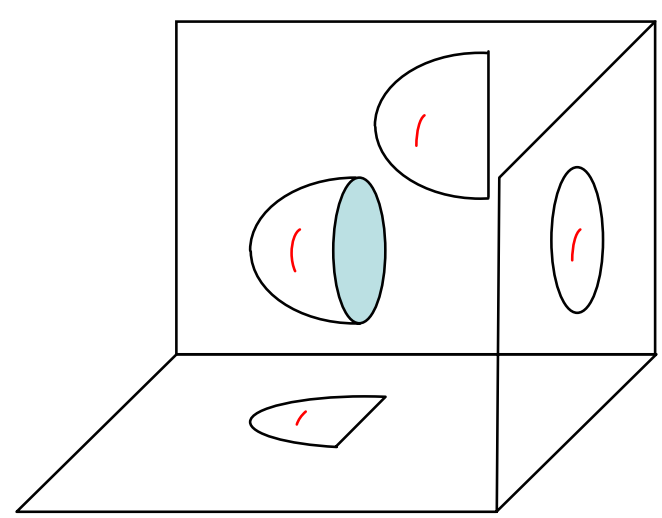

(a) Uncompressed Model

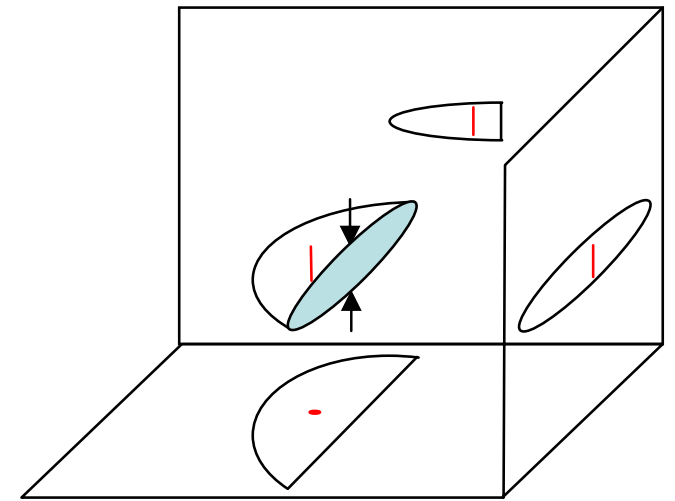

(b) Compressed Model Simulating CC view Mammogram

Figure 4. Compression in the CC View to Match Mammogram 
After the registration we back project the feature point into the 3D breast model and record the elements that correspond to that feature in X-ray CC view. These corresponding elements are aligned on a line in the compressed model and on a curve in the decompressed volume model.

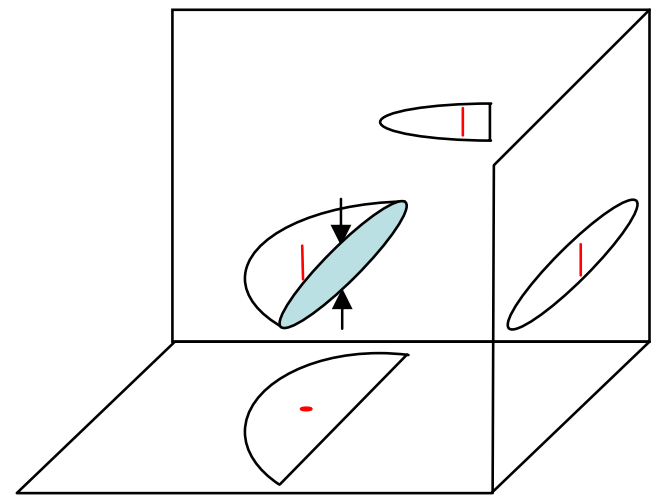

(a) Finding correspondent lesion elements in compressed model

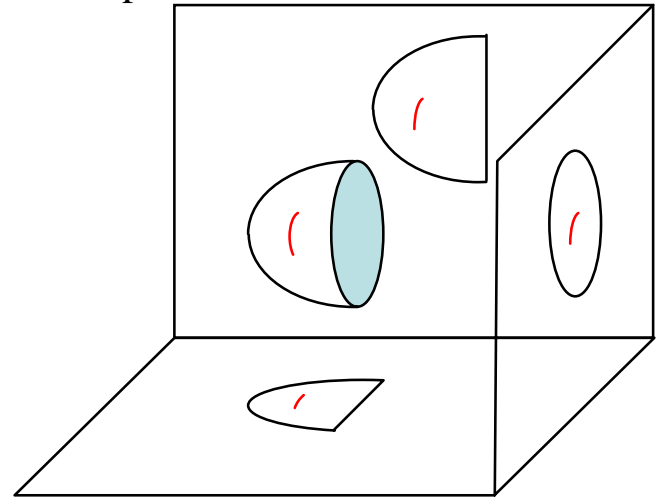

(c) Correspondent lesion elements uncompressed model

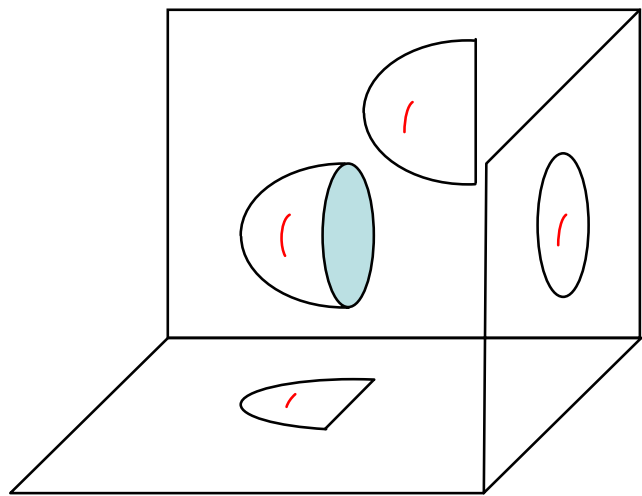

(b) Finding correspondent lesion elements in uncompressed model

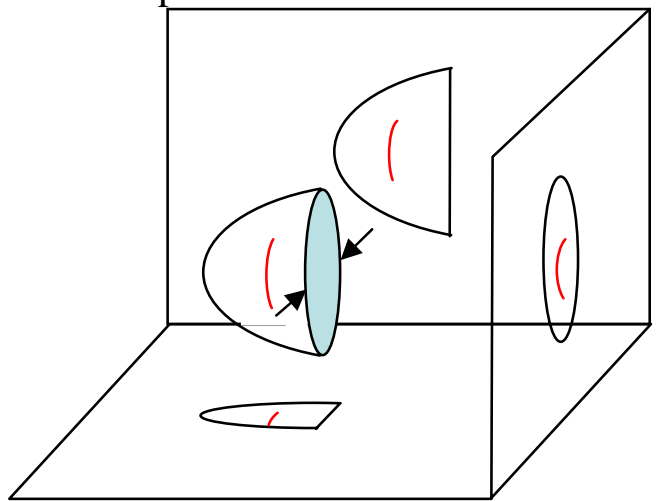

in (d) Finding Correspondent Lesion Elements In ML View

Figure 5. Finding Correspondent Lesion Elements in Two Views

The uncompressed breast model was rotated around the Oy axis with an angle equal to the angle of the X-ray ML view. Again the predicted node displacements were computed using the same solver. After model prediction, the breast model was projected again on a plane perpendicular to the compression direction and the new $2 \mathrm{D}$ projected image was registered against the ML X-ray image. The elements recorded in the CC projection lie on a 
curve in the $2 \mathrm{D}$ projected image and the feature corresponding to the same feature in the $\mathrm{X}$ ray CC view is also found on this curve.

Figure. 5. shows the process simulating mammogram compression in the ML view and predicting lesion position in ML view.

\subsection{Material Properties}

The first step toward FEM is to define the material properties for the solid model constructed in previous steps. The modeling of biomechanical tissue has gained considerable interest in a range of clinical and research applications. According to literature in the domain the breast is considered to be made of biological soft tissues, which are known to be incompressible. The female breast is essentially composed of four structures: lobules or glands, milk ducts, fat and connective tissue. The breast tissues are joined to the overlying skin by fibrous strands called Cooper's ligaments [48]. Most biologic tissues have both a viscous and an elastic response to external deformations. Because we are interested only in slow deformations the response of the tissue can be considered entirely due to elastic forces [2]. All tissues in the breast can be considered isotropic, homogenous and incompressible with nonlinear elastic properties for large deformations. The Young modulus represents how much a material will deform when a load is applied, and Poisson ratio express how much a material will shrink in one direction when is stretched in a perpendicular direction, an incompressible material will conserve the volume so same volume stretched must be shrinked. Since breast tissues exhibit a nonlinear behavior for large deformations the Young's modulus can be considered as a function of strain for each tissue: 


$$
E_{n}=\frac{\partial \sigma_{n}}{\partial \varepsilon_{n}}=b_{n} e^{m_{n} \varepsilon_{n}}
$$

where: $\sigma_{n}$ is the nominal stress for tissue type $n$,

$\varepsilon_{n}$ is the nominal strain for tissue type $n$,

$b_{n}$ and $m_{n}$ are fit parameters determined experimentally for tissue type $n$ [2].

For glandular tissue type $b_{\text {gland }}=15,100 \mathrm{~N} / \mathrm{m}^{2}$ and $m_{\text {gland }}=10.0$, for fatty tissue type $b_{\text {fat }}=4,460 \mathrm{~N} / \mathrm{m}^{2}$ and $m_{\text {fat }}=7.4$ [2]. For skin Young's modulus can be considered given by the following formula [2]:

$$
E_{\text {skin }}=a_{i} \quad(i=1 \text { to } 3)
$$

where the values for Young' modulus depend on strain as following:

$$
a_{i}= \begin{cases}3.43 \times 10^{6} N / m^{2} & 0 \leq \varepsilon_{\text {skin }} \leq 0.54 \\ 2.89 \times 10^{7} N / m^{2} & 0.54<\varepsilon_{\text {skin }} \leq 0.68 \\ 1.57 \times 10^{8} N / m^{2} & 0.68<\varepsilon_{\text {skin }}<1\end{cases}
$$

The fitted Young's modulus versus strain polynomials are as follows [38]:

$$
\begin{gathered}
E_{\text {fat }}=0.5197 \varepsilon^{2}+0.0024 \varepsilon=0.0049 \\
E_{\text {gland }}=123.8889 \varepsilon^{3}-11.7667 \varepsilon^{2}+0.6969 \varepsilon+0.0121
\end{gathered}
$$

According to experiments and comparisons presented in [48], the improvement in error registration for the overall model provided by the previous defined equations for Young's modulus over the constant value for Young's modulus is not very important as presented in Table 1, where $E_{L}$ represents error of transformation with respect to landmarks. 
The conclusion of the study in [48] is that inaccurate assumptions about the surface displacement vectors have a much larger effect on the performance of the FEM breast models than using a model with different tissue properties.

Table 1. Error Comparison for Different Models for Young's Modulus

\begin{tabular}{|c|c|c|c|c|c|c|c|c|c|c|}
\hline \multirow[t]{3}{*}{ No } & \multirow[t]{3}{*}{ Model } & \multirow{2}{*}{\multicolumn{3}{|c|}{ PCG Solver }} & \multicolumn{3}{|c|}{ Frontal Solver } & \multicolumn{3}{|c|}{$\begin{array}{c}\text { Sparse Direct } \\
\text { Solver }\end{array}$} \\
\hline & & & & & \multicolumn{3}{|c|}{$\mathrm{E}_{\mathrm{L}}[\mathrm{mm}]$} & \multicolumn{3}{|c|}{$\mathrm{E}_{\mathrm{L}}[\mathrm{mm}]$} \\
\hline & & mean & std & $\max$ & $\begin{array}{c}\text { mea } \\
n\end{array}$ & std & $\begin{array}{c}\mathrm{ma} \\
\mathrm{x}\end{array}$ & mean & std & $\max$ \\
\hline 1 & $\begin{array}{c}\text { Constant Young's } \\
\text { modulus [1KP] }\end{array}$ & 2.12 & 1.01 & 3.46 & 2.12 & 1.01 & $\begin{array}{c}3.4 \\
6 \\
\end{array}$ & 2.12 & $\begin{array}{c}1.0 \\
1 \\
\end{array}$ & 3.45 \\
\hline 2 & $\begin{array}{l}\text { Additional skin } \\
\text { [88KP] }\end{array}$ & 2.22 & 1.21 & 3.77 & 2.22 & 1.21 & $\begin{array}{c}3.7 \\
6 \\
\end{array}$ & 2.22 & $\begin{array}{c}1.2 \\
1 \\
\end{array}$ & 3.77 \\
\hline 3 & $\begin{array}{l}\text { Constant Young's } \\
\text { modulus [10KP] }\end{array}$ & 2.49 & 1.01 & 3.99 & 2.49 & 1.00 & $\begin{array}{c}3.9 \\
9\end{array}$ & 2.49 & $\begin{array}{c}1.0 \\
1\end{array}$ & 3.99 \\
\hline 4 & $\begin{array}{l}\text { Young's modulus } \\
\text { modeled (35) }\end{array}$ & 2.17 & 0.98 & 3.38 & 2.17 & 0.98 & $\begin{array}{c}3.3 \\
8 \\
\end{array}$ & 2.17 & $\begin{array}{c}0.9 \\
8 \\
\end{array}$ & 3.38 \\
\hline 5 & $\begin{array}{l}\text { Young's modulus } \\
\text { modeled (36) (37) }\end{array}$ & 2.53 & 0.85 & 3.86 & 2.53 & 0.85 & $\begin{array}{c}3.8 \\
6 \\
\end{array}$ & 2.53 & $\begin{array}{c}0.8 \\
5\end{array}$ & 3.86 \\
\hline & Max. difference & 0.41 & 0.36 & 0.53 & 0.41 & 0.36 & $\begin{array}{c}0.5 \\
3\end{array}$ & 0.41 & $\begin{array}{c}0.3 \\
6\end{array}$ & 0.54 \\
\hline
\end{tabular}

\subsection{Meshing and Discretization}

Mesh generation is one of the key components in device simulation. A quality mesh not only is necessary for obtaining good simulation results, but also has a significant impact on the computation time and efficient usage of computer resources. A mesh is a partition of geometric region into a set of non-overlapping sub-regions. Each sub-region is called an element and is characterized by its points (also called vertices or nodes), edges and faces. Mesh elements are simply connected, convex polyhedrons. The mesh process is based on the divide-and-conquer principle and involves dividing the volume in many small nonoverlapping entities called elements for which the equations (22)-(24) can be easily defined and computed. Usually these elements are tetrahedrons or hexahedrons for 3D volume 
meshing. These element types and their properties are presented in Fig. . Generally meshes with hexahedral elements are superior to ones with tetrahedral elements in terms of convergence, stability of solution in nonlinear systems and accuracy.

Many researchers had conducted studies to measure the elasticity parameters of soft tissues [42] [2] [46] [44] [47]. According to these studies the average Young' modulus value for fatty tissue is $1 \mathrm{KPa}$, for skin is $88 \mathrm{KPa}$, for glandular tissue is $10 \mathrm{KPa}$. The overall Young's modulus is considered to lie in the range 5KPa-15KPa for the entire breast modeled as a linear, continuous, incompressible, isotropic and homogenous tissue. Since we are interested in constructing a generic model we choose an initial value for Young's modulus of $10 \mathrm{KPa}$. Since the breast is considered to be an incompressible tissue, theoretically volume is preserved for a Poisson's ratio equal to 0.5. However high Poisson's ratio can lead to instabilities in FE model, a value between 0.490-0.495 is generally accepted as a computational stable and minimum displacement error for Poisson's ratio. After model calibration we used a Poisson's ratio value of 0.490 .

To mesh the solid model of the breast we used a feature first type of mesh based on Delaunay principle. The features that composed initial mesh points were the sampled border points. The mesh was composed of tetrahedral elements with 10 nodes (each side has an additional node in the middle to model the deformations more accurately)

The resulted meshed volume is presented in Figure 14. This is the finite element of the breast to which the deformations will be applied. For a 10 pixels sample interval in the original image slice, 52 slices were stacked to construct the volume and an element size of 8 units, the meshing resulted in 13225 nodes and 8744 elements, with 2 bad shape elements. Because of the complex geometry of the breast solid model and the size of the 
element resulted a small percent of bad shaped elements. This could be alleviated by further reducing the element size but this would lead to large number of elements and nodes and a long computational time. This value for element size is acceptable regarding computational time, the percent of bad shaped elements and final result error. Further reduction of the element size would have no effect on the final result. Table 2 presents the number of nodes and elements of the finite element model as a function of element size for sampling interval along the $\mathrm{z}$ axis equal to 3 . As it can be seen, the number of nodes and elements grows exponentially and so does the computational time. Table 3 presents the number of nodes and elements has the finite element model as a function of element size for sampling interval along the $\mathrm{z}$ axis equal to 1 . Increasing the sample interval on the $\mathrm{z}$ axis results in a smoothing of the breast model, value of three was used in our experiments because it was the lowest value for which the FEM was stable and number of bad shape elements minimum resulting in a robust solution.

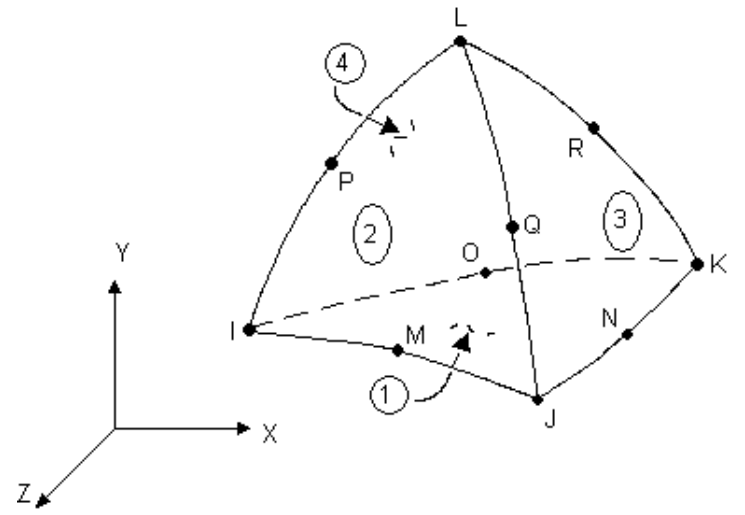

(a)Tetrahedral elements 10 nodes quadratic behavior
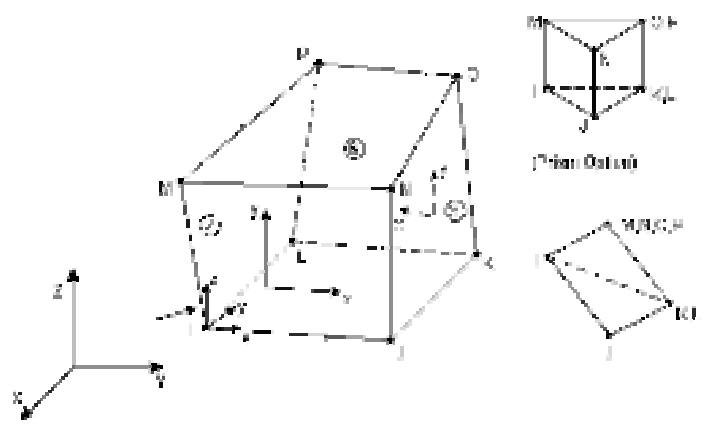

(b)Hexahedral elements 8 nodes large deformation

Figure 6. Tetrahedral Elements and Hexahedral Elements - ANSYS 


\subsection{Registration}

In order to identify the elements that correspond to a selected feature in CC image or to identify the locus of the candidate feature in the ML image, a 2D rigid registration must be accomplished between the X-ray image and the projected 3D volume.

Because of the characteristics of both images only rigid image registration is considered, which means two images can be matched through the linear transform.

The registration involves the following basic steps:

- feature-based registration;

- the 3D compressed model must be projected on a plane perpendicular to the direction of compression;

- the X-ray image must be scaled such that one pixel in both images represent the same distance;

- a geometrical transformation must be found that will map one image into another.

- bilinear interpolation applied to the transformed image because in the transformed image not all the pixels will have a correspondent in the original image.

The transformation is composed of three components: rotation, scaling and translation, which can be modeled as follows:

$$
\left(\begin{array}{l}
X_{t}^{\prime} \\
Y_{t}^{\prime}
\end{array}\right)=S\left(\begin{array}{cc}
\cos \theta & \sin \theta \\
-\sin \theta & \cos \theta
\end{array}\right)\left(\begin{array}{l}
X_{t} \\
Y_{t}
\end{array}\right)+\left(\begin{array}{l}
X_{0} \\
Y_{0}
\end{array}\right)
$$

To find the parameters of the transformation first the eigenvalues and eigenvectors will be computed for the autocorrelation matrix for both images. The autocorrelation matrix is: 


$$
M=\left[\begin{array}{cc}
\sum_{I(y, x)} x^{2} & \sum_{I(y, x)} x y \\
\sum_{I(y, x)} x y & \sum_{I(y, x)} y^{2}
\end{array}\right]
$$

Image registration is based on the alignment of the eigenvectors for (46) corresponding to two largest eigenvalues of two images. From above, we get the rotation angle.

$$
\cos \theta=\frac{V_{b}^{*} \bullet V_{t}^{*}}{\left\|V_{b}^{*}\right\| \bullet\left\|V_{t}^{*}\right\|}, \sin \theta=\sqrt{1-\cos ^{2} \theta}
$$

The equation (47) doesn't give us the direction of rotation, thus we have to determine the direction according to the sign of eigenvectors.

$$
\theta_{j}=\left\{\begin{array}{c}
a \tan \left(\frac{V \cdot y}{V \cdot x}\right) \text { if } \quad V \cdot y>0, V \cdot x>0 \\
\pi+a \tan \left(\frac{V \cdot y}{V \cdot x}\right) \text { if } \quad V \cdot y>0, V \cdot x<0 \\
\pi+a \tan \left(\frac{V \cdot y}{V . x}\right) \text { if } \quad V \cdot y<0, V . x<0 \\
2 \pi+a \tan \left(\frac{V \cdot y}{V . x}\right) \text { if } \quad V . y<0, V . x>0
\end{array}, j=\{b, t\}\right.
$$

Thus the rotation angle is $\theta=\theta_{b}-\theta_{t}$.

The translation is estimated according to the mass center of two images. The mass centers of two images $\left(\mu_{x}, \mu_{y}\right)$ are defined according to the following equation:

$$
\mu_{x}=\frac{\sum_{I(i, j) \neq 0} x}{\operatorname{count}(I(i, j) \neq 0)}, \mu_{y}=\frac{\sum_{I(i, j) \neq 0} y}{\operatorname{count}(I(i, j) \neq 0)},
$$

and thus

$$
\left\{\begin{array}{l}
X_{0}=\mu_{x}^{b}-\mu_{x}^{t} \\
Y_{0}=\mu_{y}^{b}-\mu_{y}^{t}
\end{array}\right.
$$


The similarity measure is based on the pair wise pixel comparison of the base image and aligned image. The comparison function is as follows:

$$
d(i, j)=\left\{\begin{array}{r}
1 \text { if } I_{b}(i, j) \neq I_{t}^{\prime}(i, j) \\
0 \text { if } I_{b}(i, j)=I_{t}^{\prime}(i, j)
\end{array}\right.
$$

Then the similarity measure can be defined as:

$$
\operatorname{sim}[\%]=f\left(I_{b}, I_{t}^{\prime}\right)=\frac{\sum_{i}^{N} \sum_{j}^{M} d(i, j)}{\frac{\operatorname{area}\left(I_{b}(i, j) \neq 0\right)+\operatorname{area}\left(I_{t}(i, j) \neq 0\right)}{2}}
$$

The objective of the image registration is to minimize the similarity measure. Therefore, the rigid image registration can be present as an optimization model:

$$
\min \left[f\left(I_{b}, I_{t}^{\prime}\right)\right] \quad \text { st. } I_{t}^{\prime}=\left[\begin{array}{cc}
\cos \theta & \sin \theta \\
-\sin \theta & \cos \theta
\end{array}\right] I_{t}+\left[\begin{array}{c}
X_{0} \\
Y_{0}
\end{array}\right]
$$

From the discussion above, the algorithm can be outlined in three steps:

Step 1: Initialize the system.

Step 2: While $\left(\operatorname{abs}\left(f_{n}-f_{n-1}\right)>\varepsilon\right)$ do

-Translate the image

- Rotate the image

- Dilate the image and erode the image to remove the holes generated by the transform. (or perform a bilinear interpolation)

- Calculate the similarity $f_{n}=f\left(I_{b}(n)-I_{t}^{\prime}(n)\right)$

Step 3: Output the transform and the result image 
Because the MR images imaged more of the breast than X-ray images, the projected model is larger than the corresponding X-ray image. For a correct registration process, the size of the projected model has to be reduced to the mammogram size. 


\section{Chapter 3}

\section{Algorithm and Phantom Study}

The following investigations utilized Triple Modality Biopsy Phantom. Experiments were designed to provide quantitative answers to the following questions that are often raised in two-view mammography: 1 . If suspicious features are found in two views, to what degree we can determine that they actually correspond to the same tumor? Quantitative answers were computed based on the distance between 3D curves generated from back-projection and deformation modeling, which proved the accuracy of the proposed method using breast phantom with ground truth of abnormalities. 2. If a feature is found only in the first view, what is its possible position in the second view? Suspicious area was computed in the second view through a series of projection and breast deformation modeling.

\subsection{Algorithms}

\subsubsection{Algorithm Based on Minimum Euclidean Distance}

Following algorithm is implemented for validation purpose:

1. Suspicious features are found in one mammogram (for example, ML view).

2. Compress 3D finite element breast model with recorded compression data in ML view. 
3. Each feature found in ML view will be back-projected into compressed breast model to create a straight line. Back-projection is accomplished through simple ray-tracing of the feature using known camera configuration.

4. Adaptively remesh the model elements that are adjacent to the straight lines generated in step 3.

5. Label the elements through which the straight lines pass.

6. Decompress breast model to its original shape by moving plates outwards, and the straight line will be deformed into a curve. As a result, each feature in ML view will have a corresponding 3D curve in the uncompressed breast model.

7. Repeat steps 1-6 for each feature found in CC view.

8. A feature in ML view will be paired with all features in CC view. For each pair, the distance between their 3D curves generated at step 6 will be computed. Two curves will be evenly divided into segments by _ key points (including two end points of the curve). The distance between two curves will then be computed as the sum of Euclidean distances between all key points on the curves. Each feature pair will then be ranked based on the computed distance of their 3D curves. Finally, the feature pair with the highest rank (smallest distance) will be considered as a match. In other words, they are most likely related to the same breast abnormality.

9. Repeat step 8 for each feature in ML view.

\subsubsection{Algorithm for Curve Prediction}

1. A suspicious feature is found in ML view, but not in CC view (or vice versa). 
2. Compress 3D finite element model with recorded compression data in ML view.

3. Back-project ML feature into 3D model to generate a straight line.

4. Adaptively remesh elements that are adjacent to the straight line.

5. Label the elements through which the straight line passes.

6. Decompress breast model to its original shape by moving plates outwards, and the straight line will be deformed into a curve.

7. Compress model with recorded CC data. The curve of ML feature is further deformed.

8. Project the curve onto CC view film. The projected curve in CC view indicates the most suspicious area in which a second reading is strongly recommended to see whether a featured was overlooked.

\subsection{Modeling}

\subsubsection{Image Acquisition and Data Extraction}

The phantom data consist of a set of parallel two-dimensional MR images of the phantom. The distance between slices is $2.5 \mathrm{~mm}$ and the voxel size for the MRI is 1.41x1.41x2.50mm. The extracted contour set is loaded to ANSYS FEM software package where it would then be meshed into tetrahedral structural elements. 


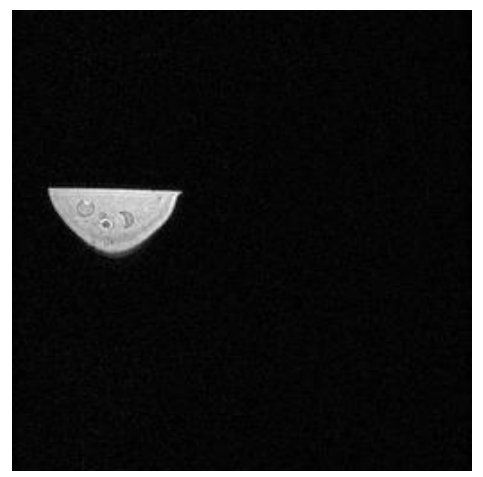

(a) Phantom MRI slice 20

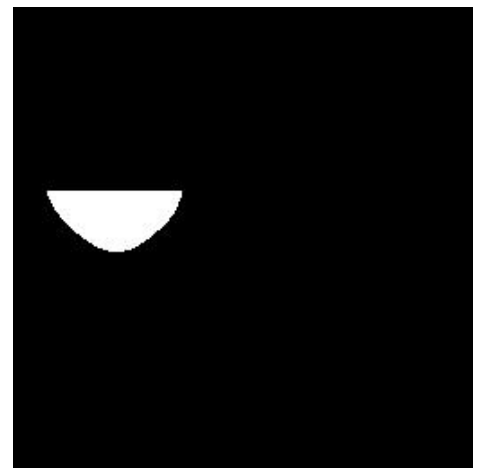

(d) Phantom MRI slice 20 segmented

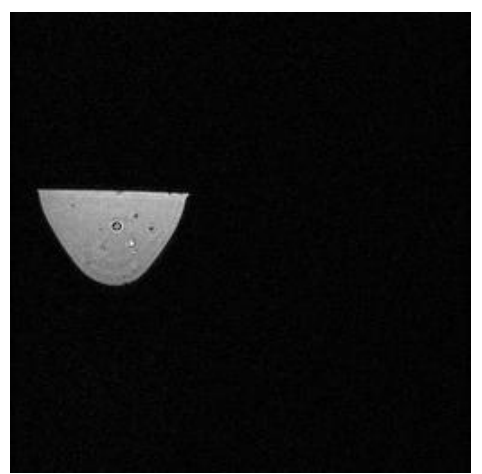

(b) Phantom MRI slice 30

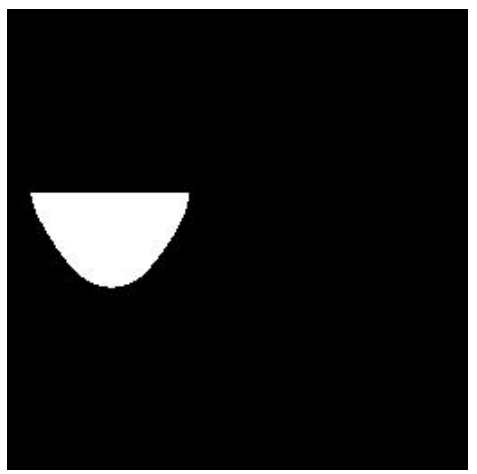

(e) Phantom MRI slice 30 segmented

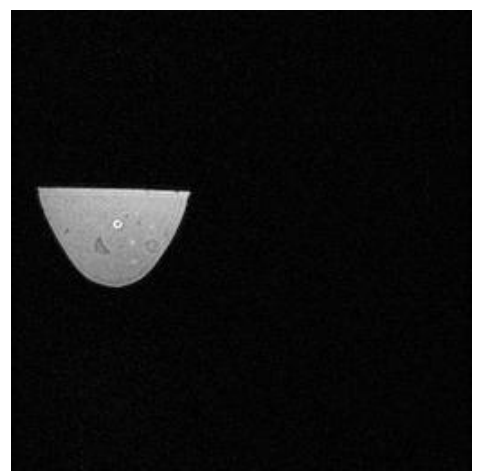

(c) Phantom MRI slice 37

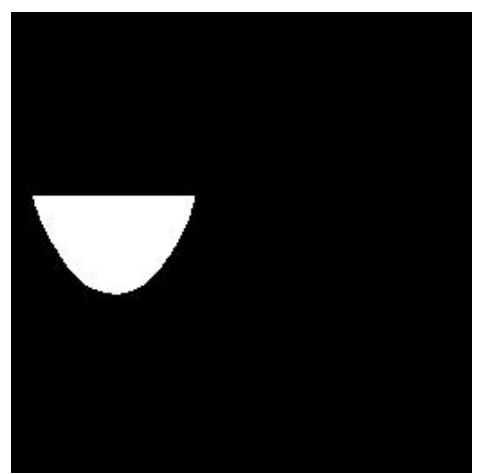

(f) Phantom MRI slice 37 segmented

Figure 7. Phantom Image Segmentation

\subsubsection{Determine Material Property}

In order to simulate breast deformations, we constructed and tested several models, each combined with different sets of simulation parameters:

1. Inhomogenous non-linear model

2. Homogenous non-linear model

3. Homogenous linear model

For example, for homogenous linear model, these tissues have been modeled for sake of simplicity as fatty tissue. The Young's modulus were set to $1 \mathrm{kPa}$ for the fatty 
tissue, and to $16.5 \mathrm{kPa}$ for the carcinoma, using published values [49]. For nearincompressibility of the tissue, the Poisson's ratio was set to 0.495 .

\subsubsection{Meshing}

Voxel- and surface-oriented meshing methods have been used to generate the meshed FEM model. The phantom volume was then meshed into isoparametric tetrahedral structural solids (elements). The elements consist of four corner nodes and an additional node in the middle of each edge. Each node has three associated degrees of freedom (DOF) which define translation into the nodal x-, y- and z-directions. Each element has a quadratic displacement behavior, and provides nonlinear material properties as well as consistent tangent stiffness for large strain applications. The skin was modeled by adding shell elements consisting of eight nodes onto the surface of the fatty tissue. Fig. 2. shows renderings of the FEM models.

Table 2. Number of Nodes and Elements

\begin{tabular}{|c|c|c|}
\hline $\begin{array}{c}\text { Element } \\
\text { size }\end{array}$ & Number of nodes & Number of elements \\
\hline 20 & 2836 & 1668 \\
\hline 15 & 4010 & 2424 \\
\hline 10 & 7899 & 5068 \\
\hline 8 & 13225 & 8744 \\
\hline 5 & 52982 & 36824 \\
\hline
\end{tabular}

The values used in our experiments were 3 for $\mathrm{z}$ axis sampling and 10 size of the element.

Since in breast model compression we do not have any information about the force applied but only about the displacement used, the boundary conditions used were Dirichelet conditions. 
Voxel- and surface-oriented meshing methods have been used to generate the meshed FEM model. The phantom volume was then meshed into isoparametric tetrahedral structural solids (elements). The elements consist of four corner nodes and an additional node in the middle of each edge. Each node has three associated degrees of freedom (DOF) which define translation into the nodal $\mathrm{x}$-, $\mathrm{y}$ - and z-directions. Each element has a quadratic displacement behavior, and provides nonlinear material properties as well as consistent tangent stiffness for large strain applications. The skin was modeled by adding shell elements consisting of eight nodes onto the surface of the fatty tissue. Figure 8. shows the renderings of the FEM models.

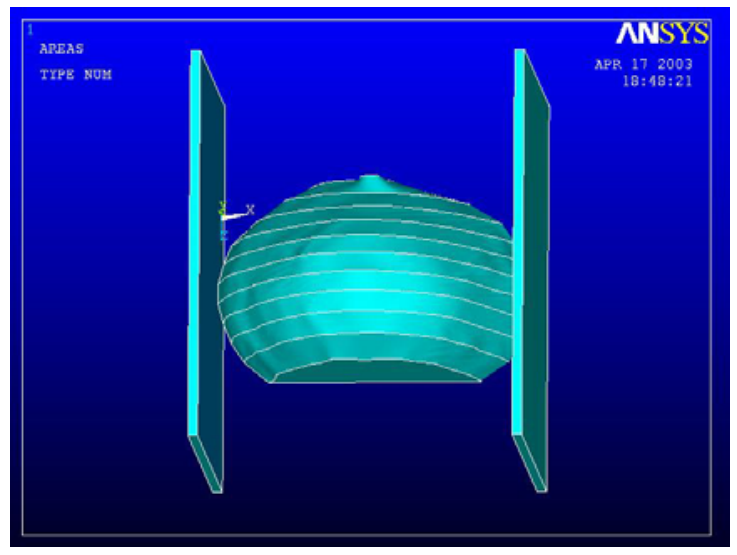

(a) Breast Volume Model

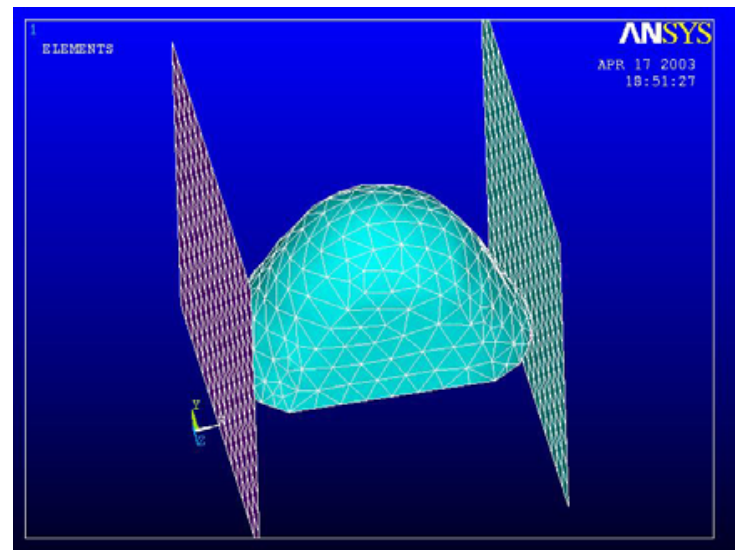

(b) Breast Model after Meshing

Figure 8. Model before and after meshing

The mesh was composed of tetrahedral elements with 10 nodes (each side has an additional node in the middle to model the deformations more accurately. The elements have a quadratic displacement behavior. The resulted meshed volume is presented in the following figure. This is the finite element of the breast to which the deformations will be applied. For a 10 pixels sample interval in the original image slice, 52 slices were stacked to construct the volume and an element size of 8 units, the meshing resulted in 4762 
nodes and 2952 elements. The number of nodes and elements grows exponentially and so does the computational time.

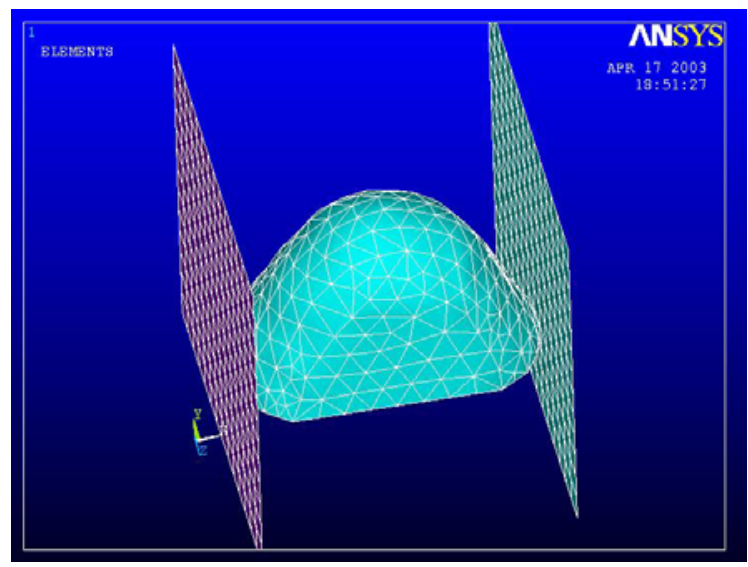

(a) FEM Breast Element Model

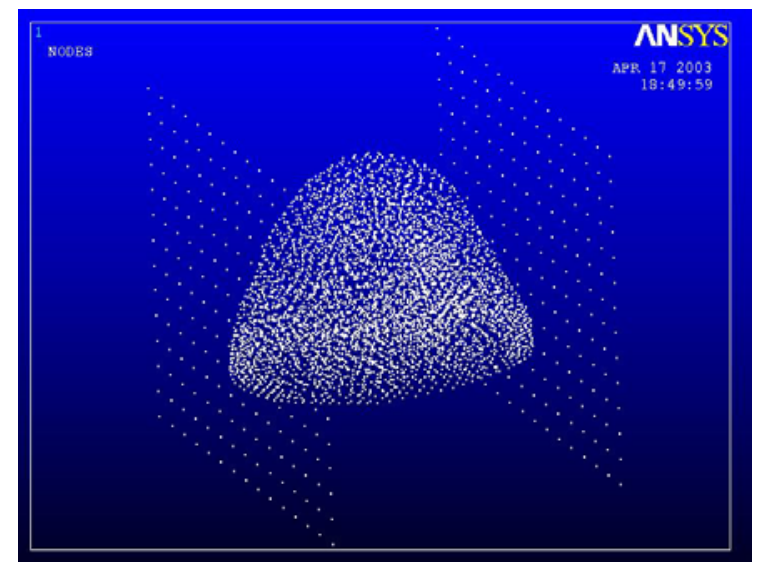

(b) FEM Breast Model Nodes

Figure 9. Finite Element Model Elements and Nodes

\subsubsection{Boundary Condition}

During X-ray imaging, force is applied through two plates that moves towards each other to compress the breast. This is a dynamic contact problem that must be simulated numerically. We will approximate breast deformation during compression by incremental stepwise simulation. The underlying assumption is that the motion of plate is slow enough so that breast deformation in each step can be described by a static equilibrium equation. More importantly, the mesh topology will not be too distorted to affect the displacement prediction. In clinical practice, the final compression magnitude is recorded, but the force exerted on plates is rarely measured. So, we will specify Dirichlet condition (displacement) on plates. To avoid sliding movement between plates and breast, we assume that once in contact with plates, the node will move only in the direction of compression. We also assign zero displacement to the nodes that lie on the ribs (chest wall). The advantage of this modeling scheme is that it can be applied to both compression and decompression, simply 
by changing directions of plate movement. The boundary condition imposed to our model is that the contact between the breast and the plates is a rough contact without any sliding, and the plates are restricted to move only along one axis.

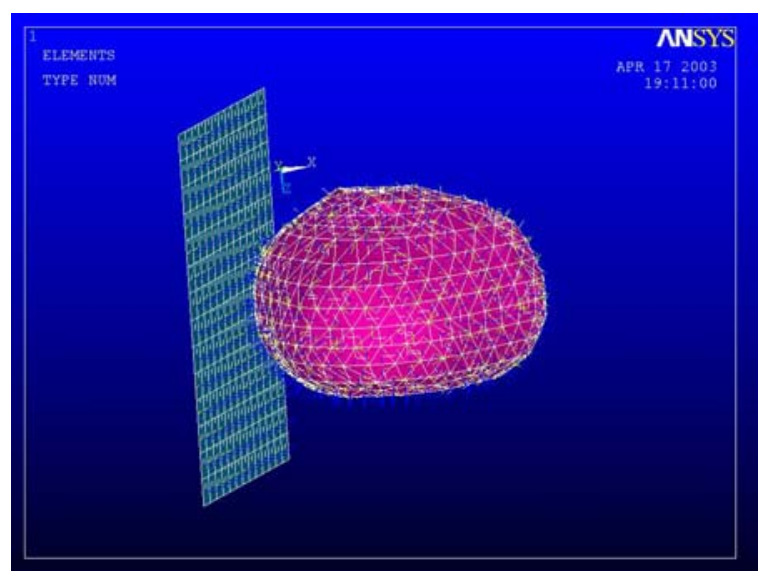

(a) Friction for left plate and model $=0.9$

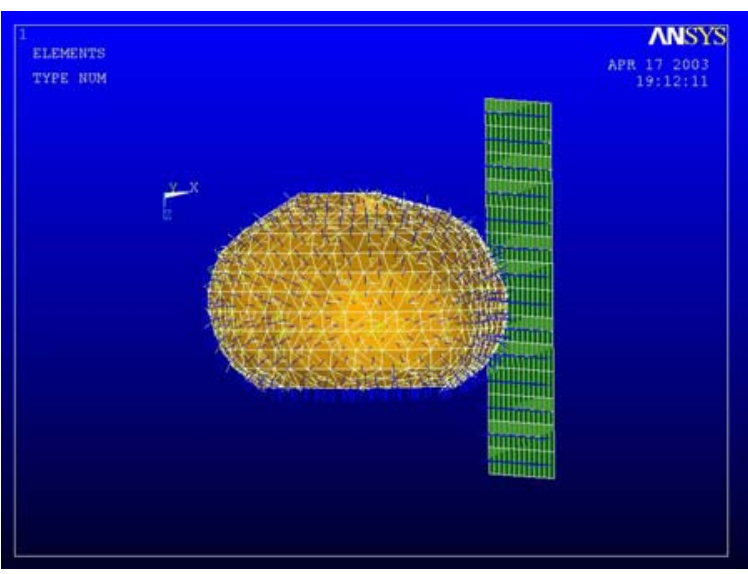

(b) Friction for right plate and model $=0.9$ Figure 10. Finite Element Model Elements and Nodes

\subsection{Compression simulation results}

To match the features between two X-ray views, similar compressions at exactly the same angle are applied to the finite element model of the breast in order to achieve similar displacements and to perform non-rigid registration between X-ray views. The compression data recorded from the $\mathrm{X}$-ray machine is as follows:

Table 3. Compression Values Used in X-ray Imaging

\begin{tabular}{|c|c|c|}
\hline $\begin{array}{c}\text { X-ray } \\
\text { view }\end{array}$ & $\begin{array}{c}\text { Compression angle } \\
\text { [degrees] }\end{array}$ & $\begin{array}{c}\text { Compression displacement } \\
{[\mathrm{mm}]}\end{array}$ \\
\hline RML & 90 & 60 \\
\hline RCC & 0 & 59 \\
\hline LML & 90 & 71 \\
\hline LCC & 0 & 60 \\
\hline
\end{tabular}


The results below (Figure 11. and Figure 12.) show the compressed model in CC view and ML view when the same displacement was added to the FEM model.

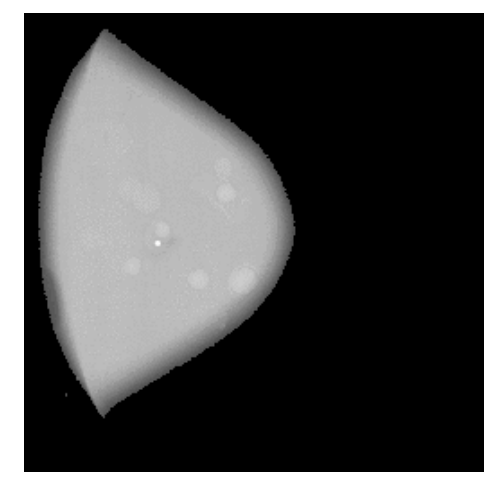

(a) Phantom CC View X-ray

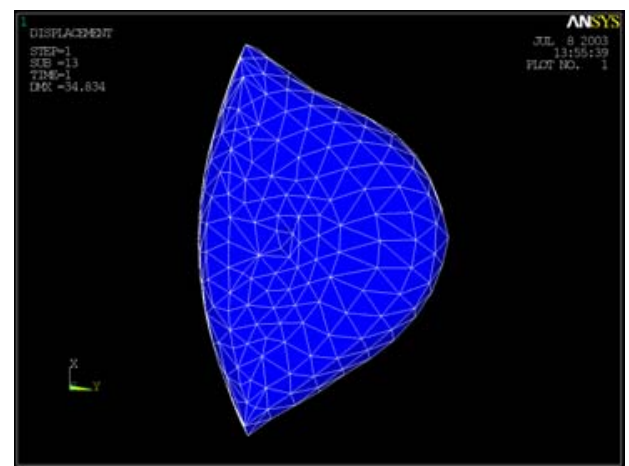

(b) Phantom model CC view after compression simulation

Figure 11. Phantom CC view compression simulation

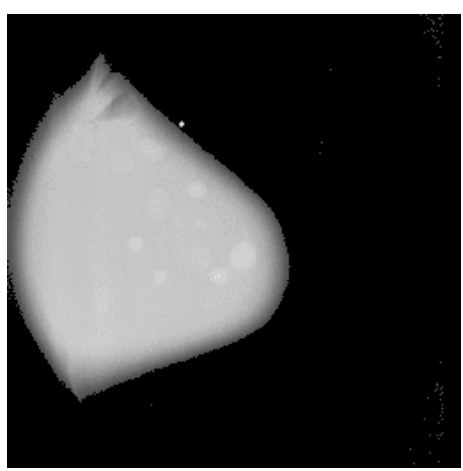

(a) Phantom ML View X-ray

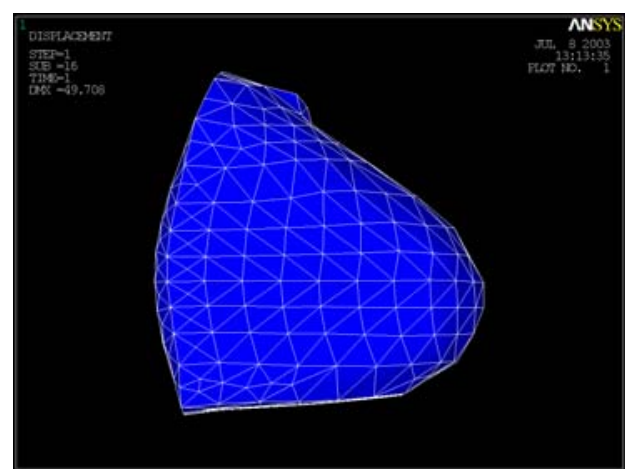

(b) Phantom model CC view after compression simulation

Figure 12. Phantom ML View Compression Simulation

\subsection{Validation}

\subsubsection{Validation Based on Minimum Euclidean Distance}

To better understand the algorithm we implemented, it is important to remember that no matter the model is compressed in which direction, when we uncompress it, the correspondent feature points in each view have always the same original position. The following procedure is repeatedly used in our experiment: we trace the original position 
first, then we combine it with the displacement to determine the feature point's final position in the correspondent view,

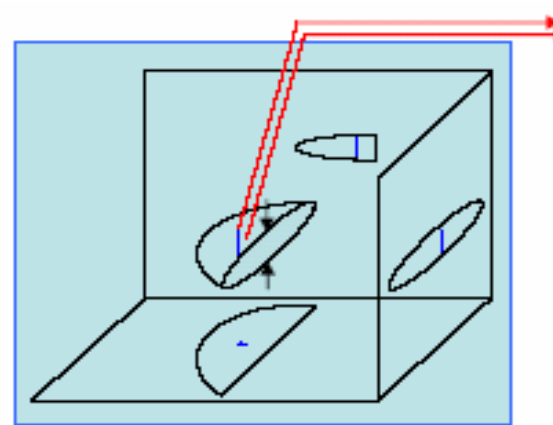

Compression CC View

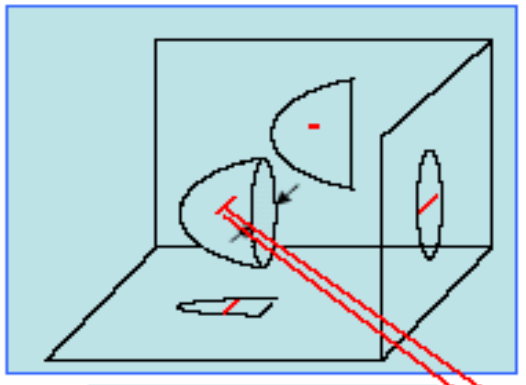

Compression ML View

\section{Nodes $(\mathrm{x}, \mathrm{y}, \mathrm{z}), \ldots$}

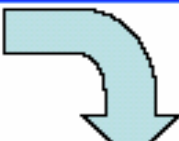

Nodes $(\mathrm{x}, \mathrm{y}, \mathrm{z}), \ldots$

Nodes $(\mathbf{x}, \mathbf{y}, \mathbf{z}), \ldots$

\section{$\operatorname{Nodes}(x, y, z), \ldots$}

Figure 13. Validation Using Minimum Euclidean Distance

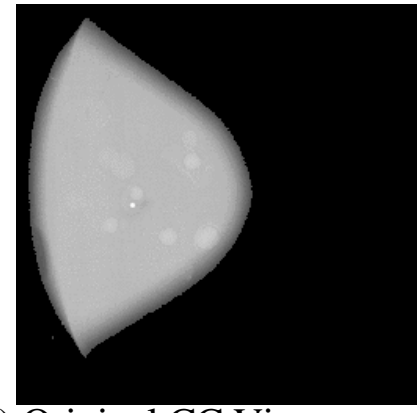

(a) Original CC View

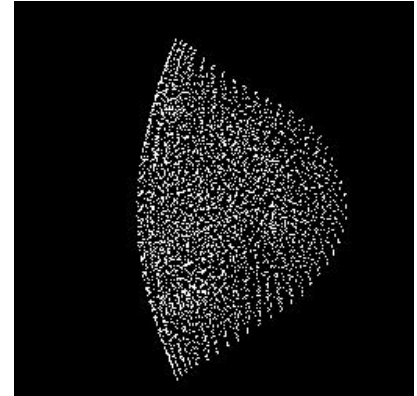

(b) Projection from Model

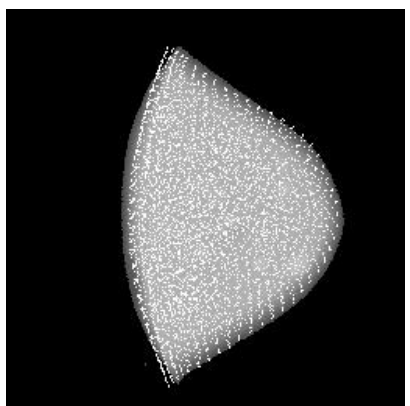

(c) Registration

Figure 14. Nodes Registered to Feature Point 
We used a small region to represent feature point 1 . Only 2952 elements were used in the model to improve the computing speed. Thus, the node's coordinate may not correspond to each single point. A correspondent point is actually a small correspondent region in our experiment.

Table 4. Process Illustration Using Node 4719

\begin{tabular}{|c|c|c|c|}
\hline \begin{tabular}{|l} 
Node \\
Number \\
\end{tabular} & $\begin{array}{l}\text { CC-X After } \\
\text { Compression }\end{array}$ & $\begin{array}{l}\text { CC-Y After } \\
\text { Compression }\end{array}$ & \\
\hline \multirow[t]{2}{*}{4719} & 79.463 & 132.515 & \\
\hline & $\begin{array}{l}\text { Displacement in } \\
\text { X Direction }\end{array}$ & $\begin{array}{l}n \text { Displacement in } \\
\text { Y Direction }\end{array}$ & $\begin{array}{l}\text { Displacement in } \\
\text { Z Direction }\end{array}$ \\
\hline \multirow[t]{2}{*}{4719} & -0.56224 & -0.98684 & -38.42 \\
\hline & $\begin{array}{l}\text { CC-X Before } \\
\text { Compression }\end{array}$ & $\begin{array}{l}\text { CC-Y Before } \\
\text { Compression }\end{array}$ & $\begin{array}{l}\text { CC-Z Before } \\
\text { Compression }\end{array}$ \\
\hline \multirow[t]{2}{*}{4719} & 80.02483 & 133.5017 & 98.22318 \\
\hline & $\begin{array}{l}\text { Displacement in } \\
\text { X Direction }\end{array}$ & $\begin{array}{l}\text { Displacement in } \\
\text { Y Direction }\end{array}$ & $\begin{array}{l}\text { Displacement in } \\
\text { Z Direction }\end{array}$ \\
\hline \multirow[t]{2}{*}{4719} & 3.2633 & 18.199 & 8.0645 \\
\hline & $\begin{array}{l}\text { ML-X Before } \\
\text { Compression }\end{array}$ & $\begin{array}{l}\text { ML-Y Before } \\
\text { Compression }\end{array}$ & $\begin{array}{l}\text { ML-Z Before } \\
\text { Compression }\end{array}$ \\
\hline \multirow[t]{2}{*}{4719} & 80.02483 & 133.5017 & 98.22318 \\
\hline & $\begin{array}{l}\text { ML-X After } \\
\text { Compression }\end{array}$ & $\begin{array}{l}\text { ML-Y After } \\
\text { Compression }\end{array}$ & $\begin{array}{l}\text { ML-Z After } \\
\text { Compression }\end{array}$ \\
\hline \multirow[t]{2}{*}{4719} & 83.28813 & 151.7007 & 106.2877 \\
\hline & $\begin{array}{l}\text { ML-X After } \\
\text { Compression }\end{array}$ & & $\begin{array}{l}\text { ML-Z After } \\
\text { Compression }\end{array}$ \\
\hline 4719 & 83.28813 & & 106.2877 \\
\hline
\end{tabular}

FEM was computed for different displacement values applied to breast model, each resulting in physically plausible displacements at each node. The method can be further developed to predict the displacement for each tissue inside the breast and for ductile tissues which have non-linear elastic and anisotropic behavior. In this study we considered only two X-ray views for matching because so it is today the screening mammography process. Because of this the feature point in the second view must be searched along a line, which represents the locus of the feature point back projected from 
the first view. Simulated calcification lies on the dotted curve. Ambiguity still exists since two calcifications are on that curve.

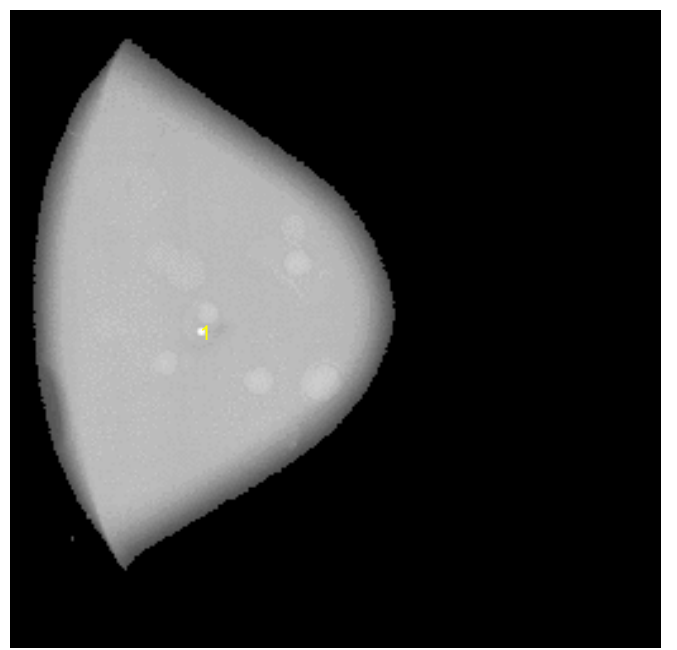

(a) Feature point 1 in CC View

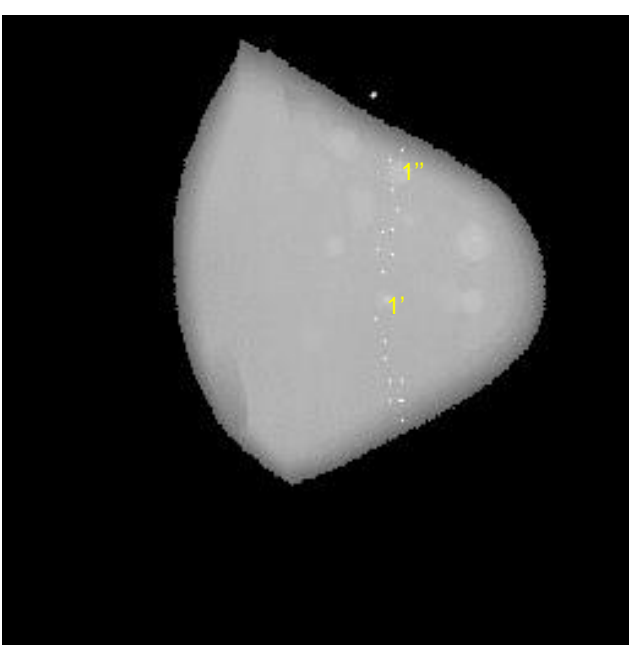

(b) Feature Point 1 in CC View Figure 15. Simulated Calcification with Predicted Curve

$\mathrm{X}$-ray mammograms of the phantom were used to evaluate the homogenous linear tissue model and the mammography compression setup. Using FEM model with the algorithm based on Minimum Euclidean Distance, we achieved the coordinates for the same lesion in the CC and ML view when the phantom was uncompressed. We calculated the Euclidean distance of all the nodes listed, generated from different views and found the minimum. The two nodes corresponding to the minimum Euclidean distance representing the lesion position in 3D coordinate system. Since each lesion position in CC view and ML correspond to a curve when we uncompress the model to the original state, the two curves would cross each other at one point, which is the lesion's exact position when the model is not compressed.

In perfect situation, the smallest distance between the curves in the uncompressed model should be zero. Table4 summarizes the accuracy of compression simulation using 
FEM deformation model. Further validation was done using MRI data. The computed coordinates from Finite Element Model for feature points were matched with coordinates calculated from MRI volume set. Distance between a feature point and its prediction:

2.6mm \pm 0.8 . Comparing with the distance between feature points: $25.9 \mathrm{~mm}-80 \mathrm{~mm}$, this result showed that the Finite Element Model can reasonably predict lesion correspondence.

Table 5. Minimum Euclidean Distance

\begin{tabular}{|c|c|}
\hline $\begin{array}{c}\text { Simulated } \\
\text { Mass and } \\
\text { Calcification }\end{array}$ & $\begin{array}{c}\text { Minimum } \\
\text { Euclidean } \\
\text { Distance (mm) }\end{array}$ \\
\hline 1 & 0.816 \\
\hline 2 & 1.134 \\
\hline 3 & 0.229 \\
\hline 4 & 1.191 \\
\hline 5 & 0.432 \\
\hline 6 & 0.824 \\
\hline 7 & 0.719 \\
\hline 8 & 1.364 \\
\hline 9 & 0.619 \\
\hline
\end{tabular}

Using generic model, we further tested on patient data with visible lesion in both CC and ML view. The minimum Euclidean distances achieved for different from patient data set were larger comparing with phantom study. The accuracy could be improved when models generated from patient MRIs were used. 


\subsubsection{Validation for Predicted Curve}

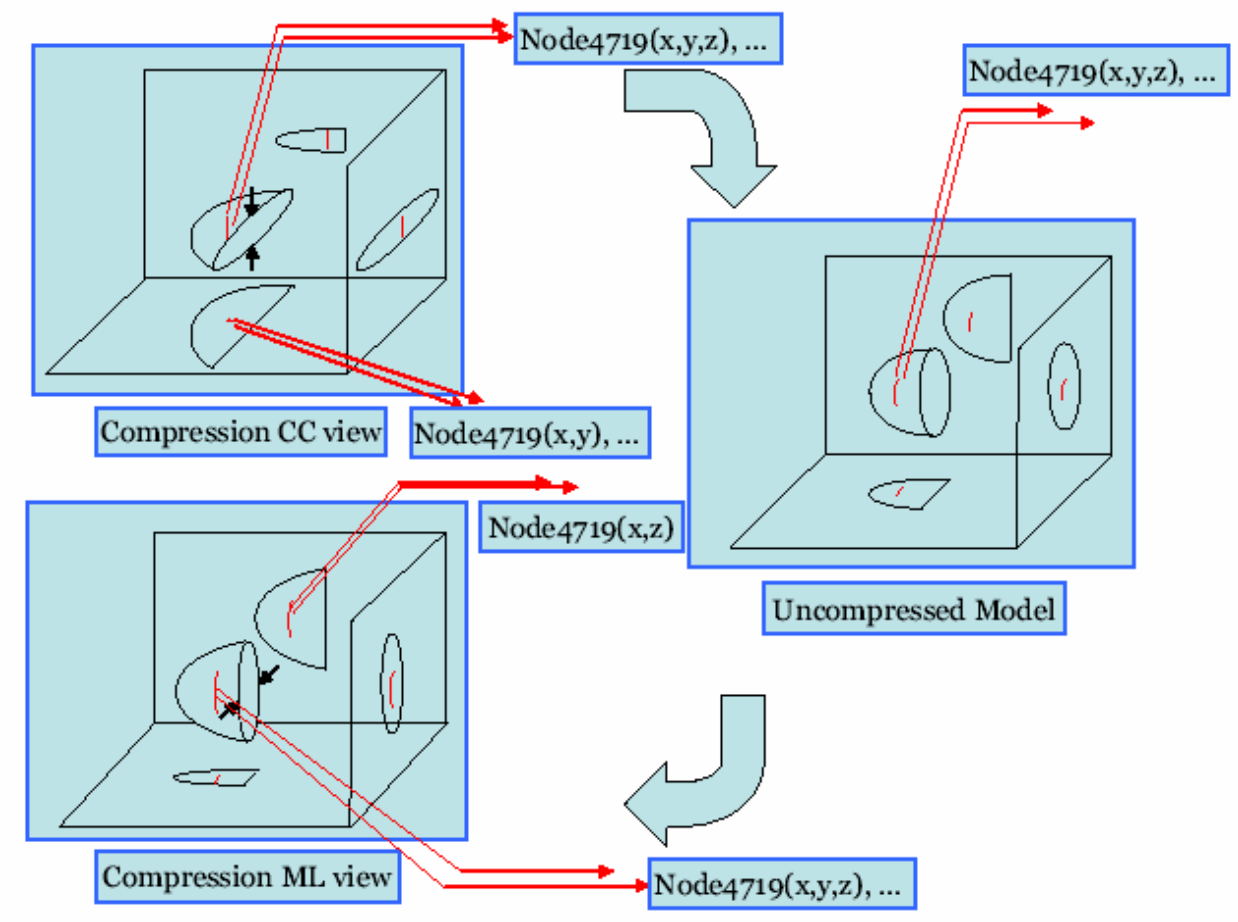

Figure 16. Validation for Predicted Curve

In cases when a suspicious feature is found in one view but not in the other view, we use FEM model to predict area in which a second reading is strongly recommended to see whether a feature was overlooked.

We tested on several cases in which lesions were visible in both views using FEM model. The results are shown as in Figure 17. The shape of the final predicted area depends on the region defined for the lesion. In the cases when the whole region of lesion was used, the resulted predicted area covered the lesion in the other view. The detailed procedure is listed below:The uncompressed breast model is compressed along the y direction with a displacement value identical to X-ray compression. After model prediction, the breast model was projected again on a plane perpendicular to the compression direction and the new 2D projected image was registered against the ML X- 
ray image. The elements recorded in the CC projection now lie on a curve in the 2D projected image and the feature corresponding to the same feature in the X-ray CC view is also found on this curve.

In order to be able to match a feature from the X-ray CC view to X-ray ML view, first the breast model is compressed along the $\mathrm{z}$ direction with a displacement value identical to X-ray compression. To solve the system of partial differential equations, a sparse method solver and a preconditioned conjugate gradient (PCG) with full NewtonRaphson iterative method were used. The PCG method performs better for solid model which has a large number of elements. After computing the predicted displacement of the nodes, the 3D compressed breast model was projected on XY plane perpendicular to the compression direction, resulting a 2D image that should match the X-ray CC view. The next step involved in experiment was to register the projected image and the CC X-ray image, matching the feature pixels from CC X-ray image to projected node positions.

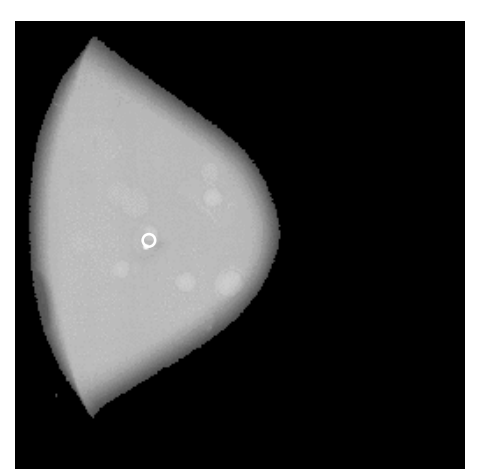

(a) Simulated Lesion in Phantom CC View

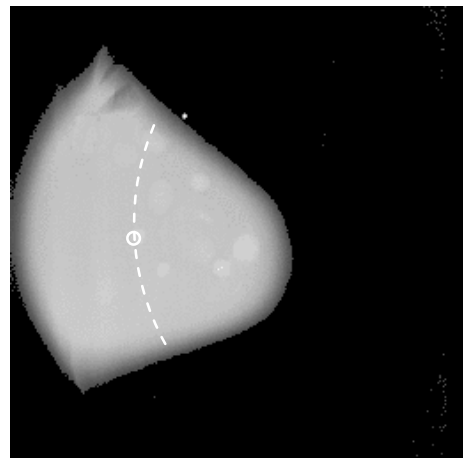

(b) Lesion Prediction in Phantom ML View Using Finite Element Model

Figure 17. Predicted Lesion Position in Phantom ML View

After the registration we back project the feature point into the 3D breast model and record the elements that correspond to that feature in X-ray CC view. These 
corresponding elements are aligned on a line in the compressed model and on a curve in the decompressed volume model.

Combining feature points' coordinates from MRI data, we further calculated the distance between the feature point and predicted curve. The minimum Euclidean distance is less than $2.1 \mathrm{~mm}$. 


\section{Chapter 4}

\section{Patient Study}

\subsection{Image Acquisition and Data Extraction}

The patient data consist of a set of parallel two-dimensional MR images of patient breasts. The distance between slices is $2.5 \mathrm{~mm}$ and the voxel size for the MRI is 1.41x1.41x2.50mm. The extracted contour set is loaded to ANSYS FEM software package where it would then be meshed into tetrahedral structural elements.

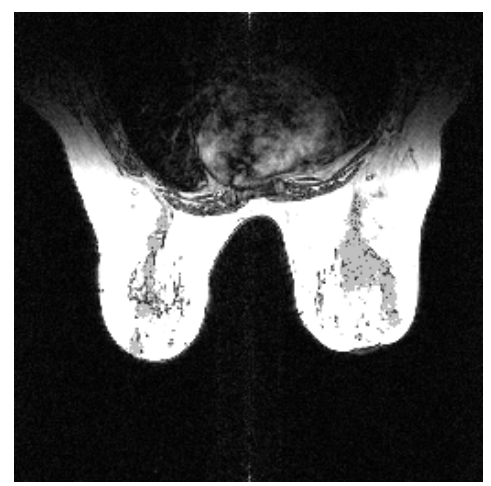

(a) Slice 28 Subject 577

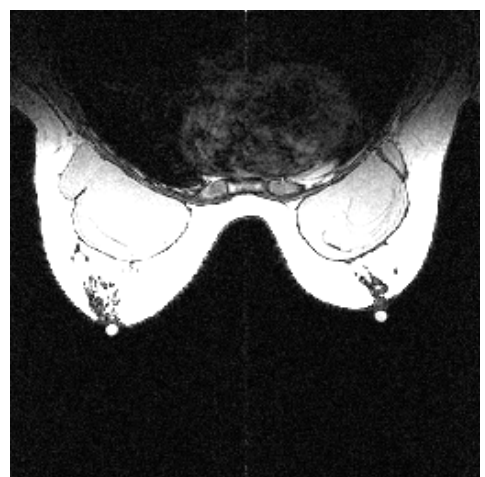

(b) Slice 34 Subject 580

Figure 18. Patient MR Images

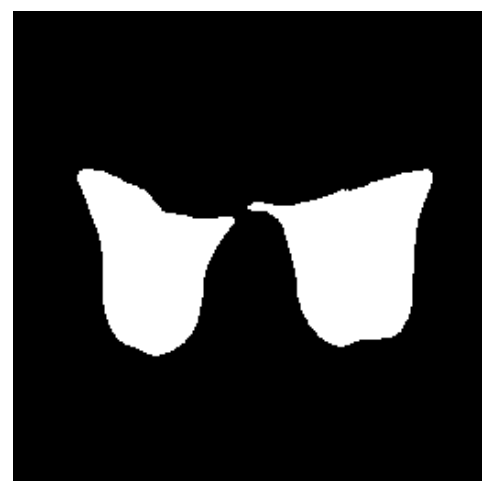

(a) Slice 28 Subject 577

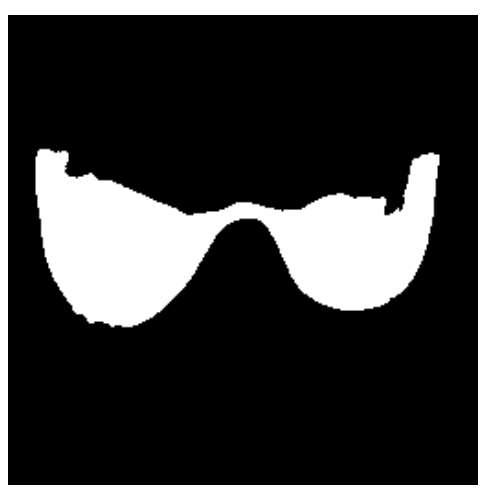

(b) Slice 34 Subject 580

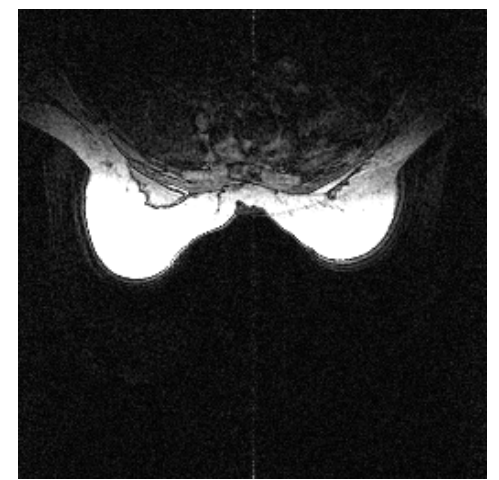

(c) Slice3 Subject 577

Figure 19. Segmented MRI Images after Morphological Operation 


\subsection{Patient Generic Model}

As similarity measures the mean and standard deviation of the Euclidian distance of corresponding landmarks were used. Landmarks were defined at prominent regions, which could be identified by physicians. In the pilot study of phantom and patient data, it was shown that a homogeneous linear elastic FEM model can best simulate the deformation of a female breast as applied during X-ray mammography.

Our goal is to generate a generic model which after scaling according to the mammogram data could be used to find correspondence in mammograms even with no MR volume.

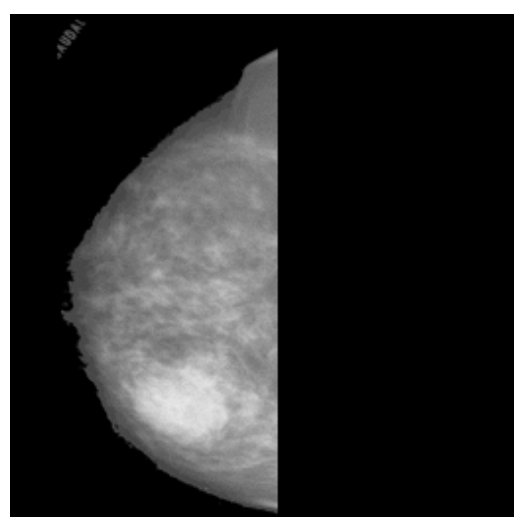

(a) CC View X-ray

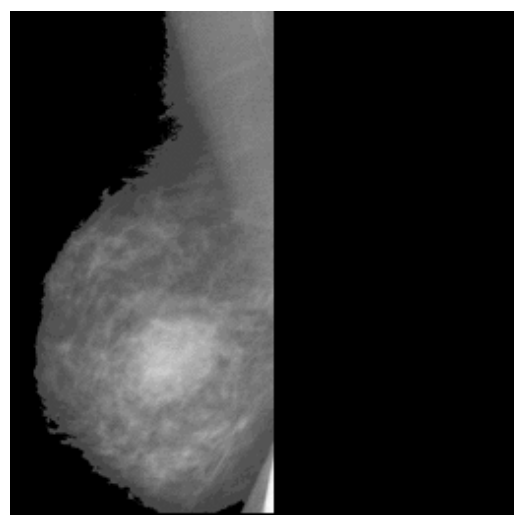

(c) ML View X-ray

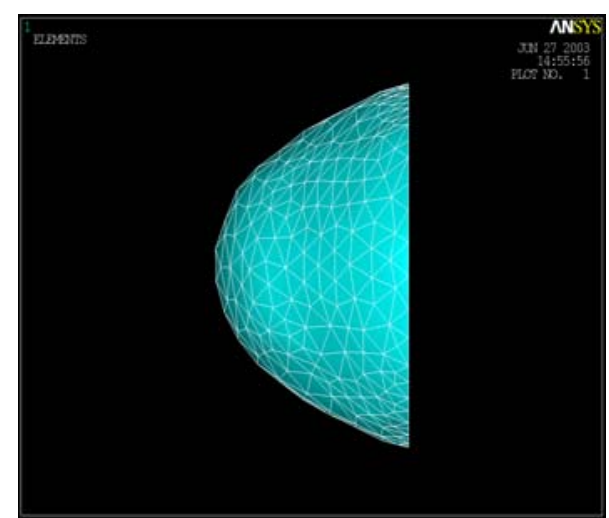

(b) Generic Model Scaled According to CC View

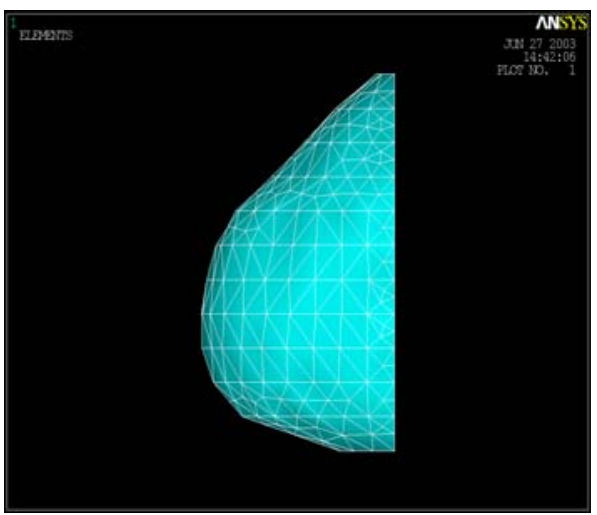

(d) Generic Model Scaled According to ML View

Figure 20. Generic Model Scaling According to CC View and ML View 


\subsection{Validation Based on Minimum Euclidean Distance}

X-ray mammograms of patients were used to evaluate the homogenous linear tissue model and the mammography compression setup. Using FEM model with the algorithm listed in Chapter 3 Phantom Study, we achieved the coordinates for the same lesion in the CC and ML view when the phantom was uncompressed. We calculated the Euclidean distance of all the nodes listed, generated from different views and found the minimum. The two nodes corresponding to the minimum Euclidean distance representing the lesion position in 3D coordinate system. Since each lesion position in CC view and ML correspond to a curve when we uncompress the model to the original state, the two curves would cross each other at one point, which is the lesion's exact position when the model is not compressed.

In perfect situation, the smallest distance between the curves in the uncompressed model should be zero. Table 6 summarizes the accuracy of compression simulation using FEM deformation model.

Using generic model, we further tested on patient data with visible lesion in both CC and ML view. The minimum Euclidean distances achieved for different from patient data set were larger comparing with phantom study. The accuracy could be improved when models generated from patient MRIs were used. 


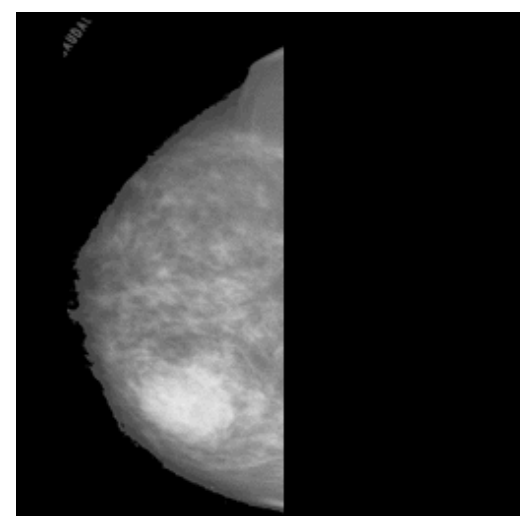

(a) Feature Point in CC View

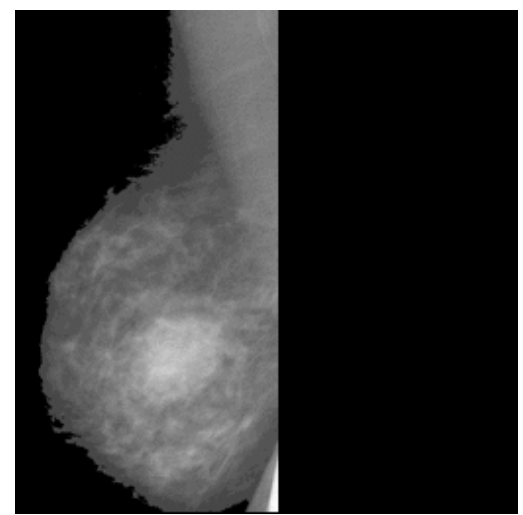

(b) Feature Point in ML View

Figure 21. Patient Data with Visible Lesion in Both Views

The minimum Euclidean distances achieved for different from patient data set were larger comparing with phantom study. The accuracy could be improved when models generated from patient MRIs were used.

Table 6. Minimum Euclidean Distance Experiment Results for Patient Data

\begin{tabular}{|c|c|}
\hline $\begin{array}{c}\text { Patient data with } \\
\text { visible lesion in } \\
\text { CC and ML view }\end{array}$ & $\begin{array}{c}\text { Minimum } \\
\text { Euclidean } \\
\text { Distance (mm) }\end{array}$ \\
\hline 1 & 1.374 \\
\hline 2 & 2.016 \\
\hline 3 & 2.349 \\
\hline 4 & 2.542 \\
\hline
\end{tabular}

\subsection{Validation for Predicted Curve}

In cases when a suspicious feature is found in one view but no in the other view, we use FEM model to predict area in which a second reading is strongly recommended to see whether a feature was overlooked.

We tested on several cases in which lesions were visible in both views using FEM model. The results are shown as in Fig. 22. The shape of the final predicted area depends on the region defined for the lesion. In the cases when the whole region of lesion was used, the resulted predicted area covered the lesion in the other view. 


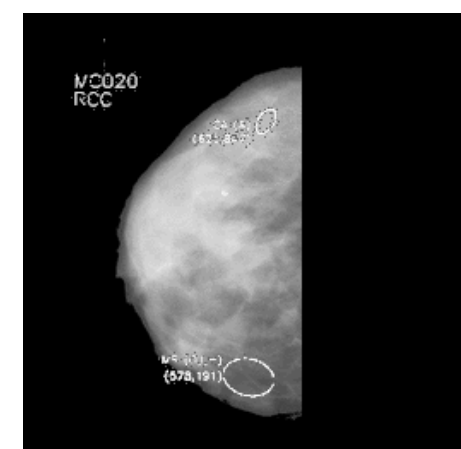

(a) Lesion in CC view

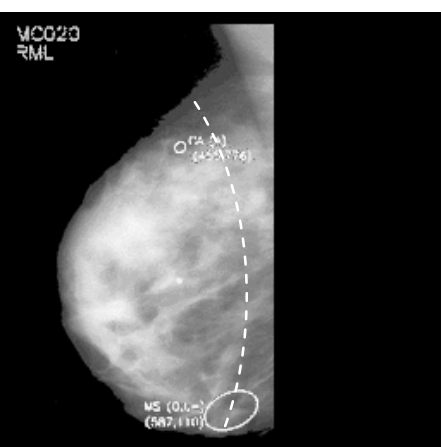

(b) Lesion prediction in ML view

Figure 22. Predicted Lesion Position in A Different View

The detail procedure is listed as follows. The uncompressed breast model is compressed along the y direction with a displacement value identical to X-ray compression. After model prediction, the breast model was projected again on a plane perpendicular to the compression direction and the new 2D projected image was registered against the ML $\mathrm{X}$-ray image. The elements recorded in the CC projection now lie on a curve in the 2D projected image and the feature corresponding to the same feature in the X-ray CC view is also found on this curve.

In order to be able to match a feature from the X-ray CC view to X-ray ML view, first the breast model is compressed along the $\mathrm{z}$ direction with a displacement value identical to X-ray compression. To solve the system of partial differential equations, a sparse method solver and a preconditioned conjugate gradient (PCG) with full NewtonRaphson iterative method were used. The PCG method performs better for solid model which has a large number of elements. After computing the predicted displacement of the nodes, the 3D compressed breast model was projected on XY plane perpendicular to the compression direction, resulting a 2D image that should match the X-ray CC view. The next step involved in experiment was to register the projected image and the CC X-ray image, matching the feature pixels from CC X-ray image to projected node positions. 
After the registration we back project the feature point into the 3D breast model and record the elements that correspond to that feature in X-ray CC view. These corresponding elements are aligned on a line in the compressed model and on a curve in the decompressed volume model. 


\section{Chapter 5}

\section{Conclusion}

Although biomechanical model has been used in breast cancer related studies [2, 21, 22, 25], to our knowledge, this is the first attempt of using a sophisticated finite element model to improve breast cancer detection with two mammographic views. Because finite element model has a unique capability of predicting nonrigid deformation accurately, a significant improvement in two-view mammographic reading can be expected.

To relieve the computational burden of finite element modeling, adaptive meshing technique will be used to reduce the element number. An incremental approach is also devised to simulate breast deformation through a series of static equilibrium computation.

We design two schemes of using $3 \mathrm{D}$ breast model to improve two-view mammographic interpretation. One scheme is for cases where both MRI and mammography are available and subject-specific model will be built from MRIs. In case that MR images are not available, we will utilizes a generic breast model to facilitate twoview mammography interpretation. This generic model-based approach is especially useful for situations where expensive MR imaging is not an affordable option.

In this work we presented a FEM for the breast, which can be successfully used in non-rigid registration. The exact values for material properties are not critical for this purpose. FEM was computed for different displacement values applied to breast model, 
each resulting in physically plausible displacements at each node. The method can be further developed to predict the displacement for each tissue inside the breast and for ductile tissues which have non-linear elastic and anisotropic behavior. In this study we considered only two X-ray views for matching because so it is today the screening mammography process. Because of this the feature point in the second view must be searched along a line, which represents the locus of the feature point back projected from the first view. In the future work more datasets from Lifetime Screening Center, Tampa FL will be combined into our work, which will be used to achieve higher accuracy of lesion correspondence analysis in breast imaging using finite element model.

$\mathrm{X}$-ray mammograms of the breast phantom and patient were used to evaluate the performance of the FE model and the described algorithm. We computed model prediction error as compared to feature size and distance of image features.

In experiments of compression simulation using FEM deformation model when suspicious area is visible in 2 views, the smallest distance between curves for 9 feature points we tested is $0.6 \mathrm{~mm}$. Ideally the smallest distance between the curves in the uncompressed model should be zero. Compared with the distance between feature points, $25.9 \mathrm{~mm}$ to $80 \mathrm{~mm}$, this result showed that the Finite Element Model can predict lesion correspondence. Validation was also performed using MRI data. The computed lesion coordinates were compared with coordinates calculated from MRI volume set. Distance between a feature point and its prediction is $2.6 \mathrm{~mm}$ based on 9 feature points in the phantom.

To validate the algorithm for cases when suspicious area is visible only in one view, a feature points visible in both views were selected. First the predicted position is 
computed (the deformed projected curve), then the minimum Euclidean distance between the real feature position and its prediction is calculated as an indicator for accuracy. The average error is less than $2.1 \mathrm{~mm}$.

Additional experiments were performed on patient breast data. 3D breast model was scaled to fit mammographic projections (just global 3D scaling). The feature points were manually identified on three sets of two view mammograms. The minimum 3D distances for projected curves were computed to validate the model and the algorithm. The average minimum distance were below $2.7 \mathrm{~mm}$, somewhat larger than in the phantom study but still very promising.

In conclusion, our initial experiments have shown that we can construct sufficiently details 3D FEM model to establish correspondences of features identified in two mammographic views. The proposed algorithm needs to be further tested and validated on larger data set. Further optimization of element sizes and meshing strategies are needed for improved accuracy. 


\section{References}

[1] Sorin, Anton, Dmitry Goldgof. “A Finite Element Model Approach in Computer Vision and it’s Application on Nonrigid Registration and Biometrics.

[2] Fred F. Azar, PhD, Dimitris N. Metaxas, PhD, Mitchell D. Schnall, MD, PhD, "A Deformable Finite Element Model of the Breast for Predicting Mechanical Deformations under External Perturbations”.

[3] AURORA, http://www.auroramri.com/redirects/idx_aurora_advantages.cfm, accessed November 1, 2003.

[4] F. S. Azar, D. N. Metaxas, and M. D. Schnall, "A Finite Model of the Breast for Predicting Mechanical Deformations during Biopsy procedure”, in IEEE Workshop on Mathematical Methods in Biomedical Image Analysis, South Carolina, USA, pp 38-45, 2000.

[5] Beek, M., Koolstra, J.H., van Ruijven, L.J., \& van Eijden, T.M.G.J. (2000), “Threedimensional finite element analysis of the human temporomandibular joint disc", Journal of Biomechanics, 33, 307-316.

[6] Stan Birchfield, "KLT: An Implementation of the Kanade-Lucas-Tomasi Feature Tracker”.

[7] Kenneth R. Castleman, “Digital Image Processing”, Prentice Hall.

[8] I. Cespedes, I. Ophir, H. Ponnekanti, and N.F. Maklad, "Elastography: Elasticity imaging using ultrasound with application to muscle and breast in vivo".

[9] Chan ,T.F. ;Vese ,L.A, “Active contours without edges”, Image Processing, IEEE Transactions on , Volume: 10 Issue: 2 , Feb. 2001 Page(s): 266 -277.

[10] Couteau, B., Payan, Y., \& Lavallee, S., "The mesh-matching algorithm: an automatic 3D mesh generator for finite element structures”, Journal of Biomechanics 2000, 33, 10051009.

[11] Dima, A.; Scholz, M.; Obermayer, K, "Automatic segmentation and skeletonization of neurons from confocal microscopy images based on the 3-D wavelet transform", Image Processing, IEEE Transactions on , Volume: 11 Issue: 7 , July 2002 Page(s): $790-801$. 
[12] J. Duncan, R. Owen, L. Staib and P. Anandan, "Measurement of non-rigid motion using contour shape descriptors”, In Proc. CVPR, June 1991.

[13] Erkamp, R.Q.; Emelianov, S.Y.; Skovoroda, A.R.; Chen, X.; O'Donnell, M, “Exploiting strain-hardening of tissue to increase contrast in elasticity imaging”, Ultrasonics Symposium, 2000 IEEE , Volume: 2 , 2000 Page(s): 1833 -1836 vol.2.

[14] J. Feldmar, N. Ayache and F. Betting, “3D-2D projective registration of free form curves and surfaces".

[15] Fuzhen Huang; Jianbo Su, "Face contour detection using geometric active contours", Intelligent Control and Automation, 2002. Proceedings of the 4th World Congress on , Volume: 3 , 2002 Page(s): 2090 -2093.

[16] Graeme P. Penney, Jurgen Weese, John A. Little, Paul Desmedt, Derek L. G. Hill, and David J. Hawkes, “A Comparison of Similarity Measures for Use in 2D-3D Medical Image Registration”.

[17] J. Hadamard, “Lectureson the Cauchy Problem in Linear Partial Differential Equations”, Yale University Press, 1923.

[18] Heath, M.; Sarkar, S.; Sanocki, T.; Bowyer, K, "Comparison of edge detectors: a methodology and initial study”, Computer Vision and Pattern Recognition, 1996. Proceedings CVPR '96, 1996 IEEE Computer Society Conference on , 1996 Page(s): 143 148.

[19] Jacob, G.; Noble, J.A.; Behrenbruch, C.; Kelion, A.D.; Banning, A.P, “A shape-space-based approach to tracking myocardial borders and quantifying regional left-ventricular function applied in echocardiography”. Medical Imaging, IEEE Transactions on , Volume: 21 Issue: 3 , March 2002 Page(s): 226 -238.

[20] F. Kallel and M. Bertrand, “Tissue Elasticity Reconstruction Using Linear Perturbation Method".

[21] Kato, J.; Watanabe, T.; Joga, S.; Rittscher, J.; Blake, A, “An HMM-based segmentation method for traffic monitoring movies”, Pattern Analysis and Machine Intelligence, IEEE Transactions on , Volume: 24 Issue: 9 , Sept. 2002 Page(s): 1291-1296.

[22] Kervrann, C.; Heitz, F.; Perez, P , "Statistical model-based estimation and tracking of nonrigid motion", Pattern Recognition, 1996., Proceedings of the 13th International Conference on , Volume: 3 , 1996 Page(s): 244 -248 vol.4.

[23] Reinhard Klette, Karsten Schluns, Andreas Koschan, “Computer Vision Three-Dimensional Data from Images”, Springer-Verlag Singapore Pte. Ltd. 1998. 
[24] Krahnstover, N.; Yeasin, M.; Sharma, R , “Towards a unified framework for tracking and analysis of human motion”, Detection and Recognition of Events in Video, 2001. Proceedings. IEEE Workshop on , 2001 Page(s): 47 -54.

[25] Kuo, C.J.; Ruey-Song Huang; Tsang-Gang Lin, “3-D facial model estimation from single front-view facial image”, Circuits and Systems for Video Technology, IEEE Transactions on, Volume: 12 Issue: 3 , March 2002 Page(s): 183 -192.

[27] Lam, S.Y; Tong, C.S., “Conformal snake algorithm for contour detection”, Electronics Letters , Volume: 38 Issue: 10,May 2002 Page(s): 452 -453.

[28] Lam, S.Y; Tong, C.S., "Fusion of physically-based registration and deformation modeling for nonrigid motion analysis”, Image Processing, IEEE Transactions on, Volume: 10 Issue: 11, Nov. 2001 Page(s): 1659 -1669.

[29] Lihong Pan; Leo Zan; Foster, F.S, “In vivo high frequency ultrasound assessment of skin elasticity”, Ultrasonics Symposium, 1997. Proceedings., 1997 IEEE , Volume: 2 , 1997 Page(s): 1087 -1091 vol.2.

[30] Lorensen, W.E. \& Cline, H.E. (1987), "Marching cubes: A high resolution 3D surface construction algorithm”, Computer Graphics, 21, 163-169.

[31] Bruce D. Lucas and Takeo Kanade, “An Iterative Image Registration Technique with an Application to Stereo Vision”, International Joint Conference on Artificial Intelligence, pages 674-679, 1981.

[32] Merickel, M.B.; Carman, C.S.; Watterson, W.K.; Brookeman, J.R.; Ayers, C.R, "Multispectral pattern recognition of MR imagery for the noninvasive analysis of atherosclerosis”, Pattern Recognition, 1988., 9th International Conference on , 1988 Page(s): 1192 -1197 vol.2.

[33] Ivana Mikic, Pamela C. Cosman, Greg T. Kogut, Mohan M. Trivedi, "Moving Shadow and Object Detection in Traffic Scenes”, ICPR 2000, Barcelona, Spain.

[34] Minolta 3-D Digitizer, http://www.minoltausa.com/vivid/products/vi900-en.asp.

[35] T. Morita and T. Kanade, “A sequential factorization method for recovering shape and motion from image streams”, IEEE Transaction on Pattern Analysis and Machine Intelligence , 19(8), pp. 858-867, 1997.

[36] M. O’Donnell, S.Y. Emelianov, A.R.Skovoroda, M.A. Lubinski, W.F. Weitzel and R.C. Wiggins, "Quantitative Elasticity Imaging”.

[37] D. Rueckert, L.I. Sonoda, C. Hayes, D.L.G. Hill, M.O. Leach, and D.J. Hawkes, "Nonrigid Registration Using Free-Form Deformations: Application to Breast MR Images”. 
[38] Abbas Samani, Jonathan Bishop, Martin J. Yaffe, and Donald B. Plewes, "Biomechanical 3D Finite Element Modeling of the Human Breast Using MRI Data”.

[39] A. Samani, J. Bishop, E. Ramsay, D. B. Plewes, "Breast Tissue Deformation Finite Element Modeling for MRI/Xray Mammography Data Fusion”.

[40] A. Samani, J. Bishop, E. Ramsay, D. Plewes, “A 3-D Contact Problem Finite Element Model for Breast Shape Deformation Derived from MRI Data”.

[41] Stan Scarloff and Alex P. Pentland, "Physically-Based Combinations of Views: Representing Rigid and Nonrigid Motion”.

[42] Julia A. Schnabel, Christine Tanner, Andy D. Castellano Smith, Martin O. Leach, Carmel Hayes, Andreas Degenhard, Rodney Hose, Derek L. G. Hill, and David J. Hawkes, "Validation of Non-Rigid Registration using Finite Element Methods".

[43] Julia A. Schnabel, Christine Tanner, Andy D. Castellano-Smith, Andreas Degenhard, Carmel Hayes, Martin O. Leach, D. Rodney Hose, Derek L. G. Hill, David J. Hawkes, "Finite element based validation for non-rigid registration using single and multilevel freeform deformations: Application to contrast-enhanced MR mammography”.

[44] J. Sciarretta, A. Samani, “Finite Element Modeling of Soft Tissues”.

[45] Shin, M.C.; Goldgof, D.; Bowyer, K.W, "Comparison of edge detectors using an object recognition task", Computer Vision and Pattern Recognition, 1999. IEEE Computer Society Conference on. , 1999 -365 Vol. 1.

[46] A. R. Skovoroda, A. N. Klishko, D. A. Gusakian, Y. I. Mayevskii, V. D. Yermilova, G. A. Oranskaja, and A. P. Sarvazyan, "Quantitative analysis of the mechanical characteristics of pathologically changed biological tissues” Biophysics, vol 40, no. 6, pp. 1359-1364, 1995.

[47] C. Tanner, A. Degenhard, J.A. Schnabel, A.D. Castellano-Smith, C. Hyes, L.I. Sonoda, M.O. Leach, D.R. Hose, D.L.G. Hill, D.J. Hawkes, "A Method for the Comparison of Biomechanical Breast Models study”.

[48] C. Tanner, A. Degenhard, J.A. Schnabel, A.D. Castellano-Smith, C. Hayes, L.I. Sonoda, M.O. Leach, D.R. Hose, D.L.G. Hill, D.J. Hawkes, "Comparison of biomechanical breast models: a case study”.

[49] C. Tomasi and T. Kanade, "Shape and motion from image streams under orthography: a factorization method”, IEEE International Journal of Computer Vision, 9(2), pp. 137-154, 1992. 
[50] Tsaig, Y.; Averbuch, A, "Automatic segmentation of moving objects in video sequences: a region labeling approach" Circuits and Systems for Video Technology, IEEE Transactions on , Volume: 12 Issue: 7 , July 2002 Page(s): 597 -612.

[51] Tsap, L.V.; Goldgof, D.B.; Sarkar, S.; Powers, P.S., “Experimental results of a vision-based burn scar assessment technique”, Biomedical Image Analysis, 1998. Proceedings. Workshop on , 1998 Page(s): 193 -201.

[52] Tsap, L.V.; Goldof, D.B.; Sarkar, S, "Nonrigid motion analysis based on dynamic refinement of finite element models", Pattern Analysis and Machine Intelligence, IEEE Transactions on , Volume: 22 Issue: 5, May 2000 Page(s): 526 -543.

[53] Leonid V. Tsap, Dmitry B. Goldgof, Sudeep Sarkar, "Fusion of Physically-Based Registration and Deformation Modeling for Nonrigid Motion Analysis", IEEE Ttransactions on Image Processing, vol.10, No. 11, November 2001.

[54] Waters, K.; Terzopoulos, D, “A physical model of facial tissue and muscle articulation”, Visualization in Biomedical Computing, 1990., Proceedings of the First Conference on , 1990 Page(s): 77 -82.

[55] Vannier, M.W.; Speidel, C.M.; Rickman, D.L.; Schertz, L.D.; Baker, L.R.; Hildebolt, C.F.; Offutt, C.J.; Balko, J.A.; Butterfield, R.L.; Gado, M.H., "Validation of magnetic resonance imaging (MRI) multispectral tissue classification”, Pattern Recognition, 1988., 9th International Conference on , 1988, Page(s): 1182 -1186 vol.2.

[56] Mingrui Zhang; Lawrence O. Hall; Dmitry B. Goldgof, "A Generic Knowledge-Guided Image Segmentation and Labeling System Using Fuzzy Clustering Algorithms", Transactions on Systems, Man, and Cybernetics - Part B: Cybernetics.

[57] C. Kambhamettu, D. B. Goldgof, D. Terzopoulos, T. S. Huang, "Nonrigid Motion Analysis", in Deformable Models in Medical Image Analysis, Chapter 19, CS Press, 1998.

[58] A. Singh, D. Goldgof, D. Terzopoulos, Editors, Deformable Models in Medical Image Analysis, IEEE Computer Society Press, 1998.

[59] C. Kambhamettu, D. Goldgof, M. He, P. Laskov, “3D Nonrigid Motion Analysis under Small Deformations", Image and Vision Computing, 21(3), pp. 229-245, 2003.

[60] Tsap, D. Goldgof, S. Sarkar, "Fusion of Physically-Based Registration and Deformation Modeling for Nonrigid Motion Analysis", IEEE Transactions on Image Processing, 10(11), pp. 1659-1669, 2001.

[61] S. Kumar, M. Sallam, D. B. Goldgof, "Matching point features under small nonrigid motion", Pattern Recognition, 34(12), pp. 2353-2365, 2001 (Honorable Mention Award by the Pattern Recognition Society). 
[62] L. Tsap, D. Goldgof, S. Sarkar, "Nonrigid Motion Analysis Based on Dynamic Refinement of Finite Element Models", IEEE Transactions on Pattern Analysis and Machine Intelligence, 22(5), pp. 526-543, 2000.

[63] L .Tsap, D. Goldgof, S. Sarkar, P. Powers, “A Vision-Based Technique for Objective Assessment of Burn Scars”, IEEE Transactions on Medical Imaging, 17(4), pp. 620-633, 1998. 